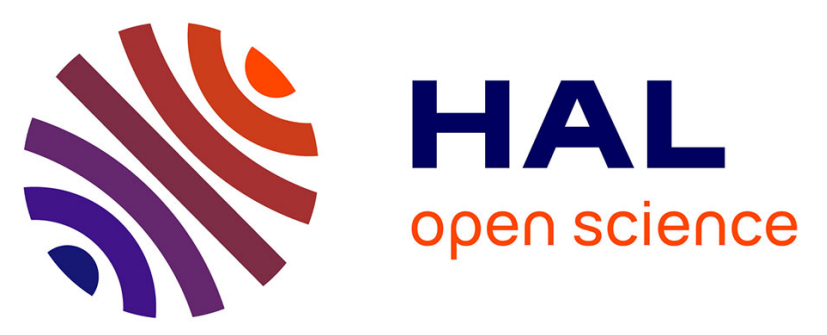

\title{
Quadruplex-interacting compounds for regulating the translation of the Epstein-Barr virus nuclear antigen 1 (EBNA1) mRNA: A new strategy to prevent and treat EBV-related cancers
}

Anton Granzhan, Rodrigo Prado Martins, Robin Fåhraeus, Marc Blondel, Marie-paule Teulade-fichou

\section{To cite this version:}

Anton Granzhan, Rodrigo Prado Martins, Robin Fåhraeus, Marc Blondel, Marie-paule Teulade-fichou. Quadruplex-interacting compounds for regulating the translation of the Epstein-Barr virus nuclear antigen 1 (EBNA1) mRNA: A new strategy to prevent and treat EBV-related cancers. Stephen Neidle. Quadruplex Nucleic Acids As Targets For Medicinal Chemistry, 54, Elsevier, pp.243 - 286, 2020, Annual Reports in Medicinal Chemistry, 978-0-12-821017-8. 10.1016/bs.armc.2020.05.001 . hal-03025274

\author{
HAL Id: hal-03025274 \\ https://hal.science/hal-03025274
}

Submitted on 7 Dec 2020

HAL is a multi-disciplinary open access archive for the deposit and dissemination of scientific research documents, whether they are published or not. The documents may come from teaching and research institutions in France or abroad, or from public or private research centers.
L'archive ouverte pluridisciplinaire $\mathbf{H A L}$, est destinée au dépôt et à la diffusion de documents scientifiques de niveau recherche, publiés ou non, émanant des établissements d'enseignement et de recherche français ou étrangers, des laboratoires publics ou privés. 


\title{
Quadruplex-interacting compounds for regulating the translation of the Epstein-Barr virus Nuclear Antigen 1 (EBNA1) mRNA: A new strategy to prevent and treat EBV-related cancers
}

\author{
Anton Granzhan, ${ }^{a, b}$ Rodrigo Prado Martins, ${ }^{c, \#}$ Robin Fåhraeus, ${ }^{c}$ Marc Blondel, ${ }^{d, e}$ Marie- \\ Paule Teulade-Fichou ${ }^{a, b}$ \\ CNRS UMR9187, Inserm U1196, Institut Curie, PSL Research University, F-91405 Orsay, France \\ CNRS UMR9187, Inserm U1196, Université Paris-Saclay, F-91405 Orsay, France \\ Inserm UMR1131, Institut de Génétique Moléculaire (IGM), Université Paris 7, Hôpital St. Louis, \\ F-75010 Paris, France \\ d Université de Brest, Inserm, EFS, UMR1078, GGB, F-29200 Brest, France \\ e CHRU Brest, service de génétique clinique et de biologie de la reproduction, F-29200 Brest, France \\ \# Current address: INRAE, Université de Tours, ISP, F-37380, Nouzilly, France
}

\begin{abstract}
The Epstein-Barr (EBV) virus is linked to at least $1 \%$ of cancers that include Burkitt's and Hodgkin's lymphomas, nasopharyngeal carcinoma, and $10 \%$ of gastric cancers. EBV is a latent virus that possesses a genome maintenance protein EBNA1, which is both essential for the virus and highly antigenic. Hence, EBV evolved a mechanism by which EBNA1 self-inhibits the translation of its own mRNA, thereby minimizing the production of EBNA1-derived antigenic peptides. Although not fully elucidated, this mechanism involves the Gly-Ala-rich (GAr) motif of EBNA1, encoded by a G-repeatcontaining mRNA sequence able to form clusters of G-quadruplexes. This chapter summarizes recent significant advances in understanding of this phenomenon. Mechanistic investigations based on yeast chemical genetics, cellular assays and in vitro experiments have shown that the host cell factor nucleolin (NCL) is involved in this limitation of EBNA1 translation through binding to G-quadruplexes of EBNA1 mRNA. This interaction can be disrupted by the benchmark G4-ligand PhenDC3 acting as NCL competitor for binding to G4-RNA. Finally, exploration of the chemical space around PhenDC3 using combinatorial chemistry approach led to the generation of 20 compounds based on a bis(acylhydrazone) scaffold. Amongst these, two hits (PyDH2, PhenDH2) exhibit optimized properties with regard to the disruption of NCL/G4 interaction in cells, along with lower cytotoxicity. Consequently, treatment by PyDH2 or PhenDH2 increases EBNA1 production and stimulates the GArrestricted antigenic response. Altogether, this innovative concept of antigenic stimulation sets the basis for further identification of lead candidates that may become promising candidate drugs for treating EBV-related cancers.
\end{abstract}

Keywords: Epstein-Barr virus (EBV), EBV-related cancers, G-quadruplex RNA, Nucleolin, Antigenic stimulation, G4-ligands 


\section{Contents}

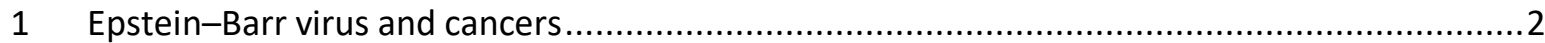

2 Epstein-Barr virus Nuclear Antigen 1 (EBNA1): the Achilles' heel of EBV ..............................5

3 G-Quadruplexes in the GAr-encoding sequence of EBNA1 mRNA ........................................7

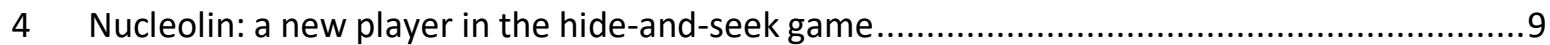

5 PhenDC3, a benchmark G4 ligand, disrupts NCL-EBNA1 mRNA interaction, enhances EBNA1 expression, and overcomes the immune evasion of EBV .........................................................14

6 Development of a novel series of G4-interacting ligands disrupting the NCL-G4 EBNA1

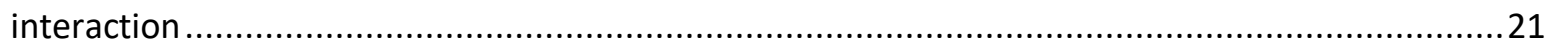

7 Conclusions

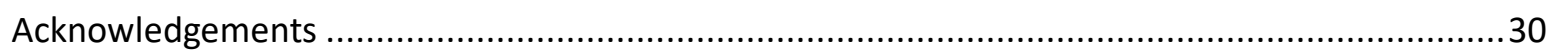

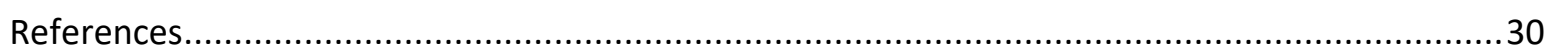

\section{Epstein-Barr virus and cancers}

The Epstein-Barr virus (EBV) belongs to the gamma-herpesvirus family (Gammaherpesvirinae) and is the first virus that has been isolated from a human cancer. ${ }^{1}$ It is one of the most ancient viruses, and infects more than $95 \%$ of the human population worldwide. Human B-lymphocytes are the primary target of EBV infection, but other cell types (mainly, epithelial cells) can also be infected. Like other herpesviruses, EBV is a double-stranded DNA virus and, after infecting the cells, establishes a latent lifecycle with its genome existing as circular genetic elements (called episomes) inside the host cell's nucleus. Viral episomes are produced in multiple copies that are closely associated to the host DNA whilst not being integrated, and thereby remains extrachromosomal. ${ }^{2}$ EBV genome is composed of approximately $180 \mathrm{~kb}$, which encode about 80-85 proteins. EBV infection persists as a lifelong, latent asymptomatic infection, indicating a fine balance between virus infection, the host cell and the host immune system. ${ }^{3}$ Periodically, the virus may become reactivated through mechanisms that are currently unclear; the viral and immune factors that determine the outcome of infection remain poorly understood. Most infections take place at a young age and are asymptomatic, but from adolescence and onwards it may induce a number of immune disorders and diseases including infectious mononucleosis (the "kissing disease"), lymphomatoid granulomatosis, and multiple sclerosis. Moreover, under certain conditions that are still unknown, the virus may cause some human cancers, a reason for which the World Health Organization defined EBV as a class I carcinogen. Some cancers are $100 \%$ associated with EBV, such as the endemic forms of Burkitt's lymphoma (BL) and the nasopharyngeal carcinoma (NPC). In other cases, such as Hodgkin's lymphoma, the virus is detected in about $50 \%$ of tumor cells. Altogether, at least $1 \%$ of cancers worldwide are estimated to be linked to EBV. ${ }^{4}$ The incidence and characteristics of these EBV related cancers are detailed below (Table 1 ). 
Table 1. EBV-associated cancers and their main characteristics: cell origin, geographic distribution, expression of viral proteins during latency phases (adapted from Ref. ${ }^{5}$ ).

\begin{tabular}{|c|c|}
\hline Disease & Characteristics \\
\hline Burkitt's lymphoma (BL) & $\begin{array}{l}\text { - B-cell origin } \\
\text { - Endemic form in Africa is } 100 \% \mathrm{EBV} \text {-associated } \\
\text { - Only EBNA1 latent protein is expressed (latency I) }\end{array}$ \\
\hline Hodgkin's lymphoma (HL) & $\begin{array}{l}\text { - } \text { B-cell origin } \\
\text { - Common in western countries (USA and Europe) } \\
\text { - } 40 \% \text { EBV-associated } \\
\text { - } \text { EBNA1, LMP1 and LMP2A/B latent proteins are expressed } \\
\text { (latency II) }\end{array}$ \\
\hline $\begin{array}{l}\text { Lymphomas in immunosuppressed } \\
\text { individuals (AIDS, post-transplant } \\
\text { patients) }\end{array}$ & $\begin{array}{l}\text { - B-cell or T-cell origin } \\
\text { - } \quad \text { EBNA1, EBNA2, EBNA3A/B/C, EBNA-LP, LMP1 and } \\
\text { LMP2A/B latent proteins are expressed (latency III) }\end{array}$ \\
\hline Nasopharyngeal carcinoma & $\begin{array}{l}\text { - } \text { Epithelial cell origin } \\
\text { - } \text { Common in south-east China and in North Africa } \\
\text { - } 100 \% \text { EBV-associated } \\
\text { - } \text { EBNA1, LMP1 and LMP2A/B latent proteins are expressed } \\
\text { (latency II) }\end{array}$ \\
\hline $\begin{array}{l}\text { T/NK cell lymphomas (nasal T/NK } \\
\text { lymphoma, aggressive NK-cell } \\
\text { leukemia, T cell lymphoproliferative } \\
\text { disorder of childhood) }\end{array}$ & $\begin{array}{l}\text { - T- or NK-cell origin } \\
\text { - Common in Asian and Latin American countries } \\
\text { - EBNA1, LMP1 and LMP2A/B latent proteins are expressed } \\
\text { (latency II) }\end{array}$ \\
\hline Gastric carcinoma & $\begin{array}{l}\text { - } \text { Epithelial cell origin } \\
\text { - } 10 \% \text { EBV-associated } \\
\text { - } E B N A 1, \text { LMP1 and LMP2A/B latent proteins are expressed } \\
\text { (latency II) }\end{array}$ \\
\hline
\end{tabular}

- Burkitt's lymphoma (BL) is the first cancer that has been linked to EBV infection. BL are classified into three groups, namely endemic (40-45\%), sporadic (5-15\%), and HIV-associated forms (40\%). More than $90 \%$ of endemic BL are EBV-positive, and all are characterized by the chromosomal translocation of the c-Myc locus from its normal localization on chromosome 8 to one of the Immunoglobulin (Ig) heavy or light chain loci located on chromosomes 14,2 , or 22 respectively.

- Hodgkin's Lymphoma (HL) is one of the most frequent lymphomas in the Western world, where it constitutes 20 to $50 \%$ of all lymphoma cases. HL can be divided into lymphocytepredominant ( $\mathrm{LP}-\mathrm{HL})$ and classical $(\mathrm{CHL})$, including mixed cellularity, nodular sclerosis and lymphocyte-rich subtypes. About $40 \%$ of $\mathrm{HL}$ cases are EBV-infected. Clonal viral genomes can be found in all of the tumor cells, and the virus remains in malignant cells during the whole course of disease. Typically, most $\mathrm{HL}$ cells present a deregulation of B-cell specific genes and show a strong expression of the antiapoptotic factor NF-kB which contributes to the survival of cancer cells. 
- T-cells and Natural Killer (NK)-cells lymphomas are rare, aggressive non-B-cell tumors that occur primarily in Asia and Latin America, but are comparatively uncommon in other areas. Activation of NF-kB is also typical of these cancers.

- Post-transplant lymphomas (PTL) present high risk for T-cell immunocompromised patients (mostly, after transplantation). Most post-transplant lymphomas have an early onset, typically within the first year of allografting, and almost all of these tumors are EBV-positive.

- Nasopharyngeal carcinoma (NPC) is a rare epithelial head and neck cancer with an incidence of about 1 per 100,000 per year in most parts of the world. However, NPC is very common in Southern China and in Tunisia where its prevalence is $25-30$ cases per 100,000 people every year and represents one of the most predominant cancers among men (mostly of 40-60 years old). All NPC are associated with EBV. NPC is highly aggressive with an extensible local infiltration and early metastasis.

- Gastric carcinomas: Between 2 and $20 \%$ of gastric cancers are associated with EBV infection in epithelial cells, with a world average of $10 \%$. It is most common in men and the predominant localization is the proximal stomach. The most common factor associated to gastric cancers is Helicobacter pylori infection. However, H. pylori infection is not a risk factor for gastric carcinoma associated to EBV, which suggests that both pathogens are involved in different carcinogenic pathways.

Treatment of EBV related cancers. A number of pharmacological and immunotherapeutic approaches are being developed to treat, or prevent, EBV-associated tumors. However, more than 50 years after the identification of EBV as an oncogenic virus, there is still no specific treatment against EBV-related cancers and others EBV-related diseases. As for cancers, the treatment relies on the use of radiation, classical polychemotherapy (anthracyclines, cylophosphamide, vincristine), and immunotherapy using monoclonal antibodies (Rituximab) either alone or in combination. Regarding EBV-related lymphoma, there are only few therapies specifically targeting the latent virus but, in most cases, the available treatments are the same than the ones used for EBV-negative lymphomas and are mainly based on adoptive immunotherapy (i.e., infusion of cytotoxic T-cells). This therapy has proven efficacy for treating post-transplant lymphomas (PTL) that arise in particular immunodepressed context. However, this is not applicable to EBV-related tumors in immunocompetent individuals who show more restricted viral gene expression and thus less immunogenicity. ${ }^{6}$

Of note, the expression profiles of EBV viral proteins are highly dependent on the type of EBV-cancer (Table 1), which may strongly modulate the effects of antiviral therapies based on anti-herpesvirus agents like Acyclovir or Ganciclovir. Indeed, these drugs are DNA polymerase inhibitors, but replication of latent EBV in proliferating $B$ cells does not require the viral polymerase and therefore antiviral therapy is usually ineffective. In addition, Ganciclovir, a guanosine analogue inhibiting the viral replication, requires phosphorylation to become active, while viral kinases are typically not expressed in EBV-related tumors.

Finally, production of an effective vaccine for EBV have not yet been successful despite significant efforts towards production of recombinant EBV proteins in yeast. ${ }^{7,8}$ This is most certainly due to the very complex lifecycle of the virus and to the deeply different immunogenic and viral expression patterns of the EBV-derived cancers. For all these reasons, there is a strong need to develop new therapies exploiting specific mechanisms of EBV biology, which should provide targeted strategies for EBV-related cancers. In particular, novel strategies based on molecular mechanisms that provide viral 
antigenic peptides and on viral factors controlling the switch from latent to lytic phases currently represent new active research areas in the field. ${ }^{5}$

\section{Epstein-Barr virus Nuclear Antigen 1 (EBNA1): the Achilles' heel of EBV}

The life cycle of EBV comprises two major phases: the latent phase and the lytic phase. During the latent phase, EBV has a strict gene expression pattern which includes six EBV nuclear antigens (EBNAs), two latent membrane proteins (LMPs), as well as EBV-encoded small RNAs (EBERs) and miRNAs (BART). Depending on the proteins expressed, EBV latent infection can be classified in three different categories (Latency I, II and III, Table 1). Despite their differences, all latency programs have in common the production of EBV Nuclear Antigen 1 (EBNA1), the genome maintenance protein (GMP) of EBV that is required for both replication and mitotic segregation of the episome during host cell division (hence its name). Therefore, the presence of EBNA1 protein in all EBV-carrying tumors constitutes a hallmark that distinguishes the virus-associated cancer cells from normal cells and thereby offers therapeutic opportunities for targeted interventions.

EBNA1 is a multifunctional protein that exerts essential functions in viral DNA replication and episome maintenance. Indeed, EBNA1 is required to ensure the extrachromosomal replication of viral episomes and mediates their segregation during cell division. EBNA1 also interacts with viral and cellular promoters contributing to the transcriptional regulation of both viral and cellular genes, directly or indirectly. EBNA1 is a dimeric protein containing three different functional domains: a central glycinealanine (GAr) domain, two arginine-glycine (RG) domains (called linking regions, LR1 and LR2), and a Cterminal DNA-binding domain (DBD) involved inter alia in the association of the viral genome to the host cell chromatin (Figure 1). ${ }^{9,10}$ Interestingly, LR1 and LR2 domains of EBNA1 are known to interact with G-quadruplex RNA structures (including those formed in its own mRNA, vide infra), and this interaction seems to play a critical role in recruiting the cellular origin recognition complex (ORC), ensuring the episome maintenance. Moreover, quadruplex-interacting drugs such as BRACO-19, TMPYP3 and TMPyP4 disrupt the interaction of EBNA1 with G4-RNA and interfere with recruitment of ORC, thereby inhibiting viral replication. Thus, treatment of EBV-infected Raji cells with BRACO-19 results in a moderate reduction of EBV copy number and selective death of EBV-positive cells, providing a first example of the use of a quadruplex-mediated strategy for the development of targeted therapy against EBV. ${ }^{10}$ 
$\mathrm{N}$

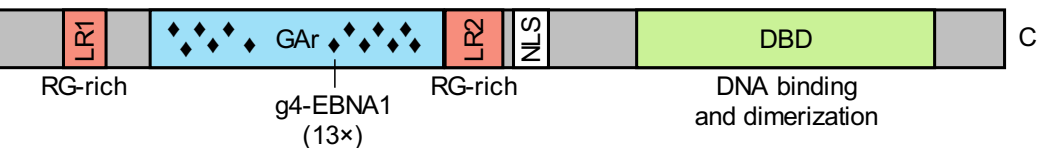

EBNA1 $\triangle$ GAr

$\mathrm{N}$

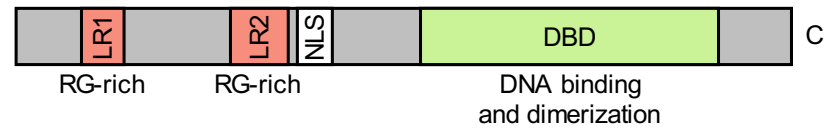

B

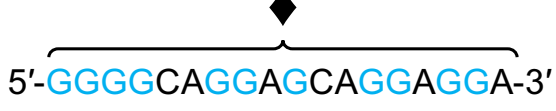

repeat motif (g4-EBNA1)

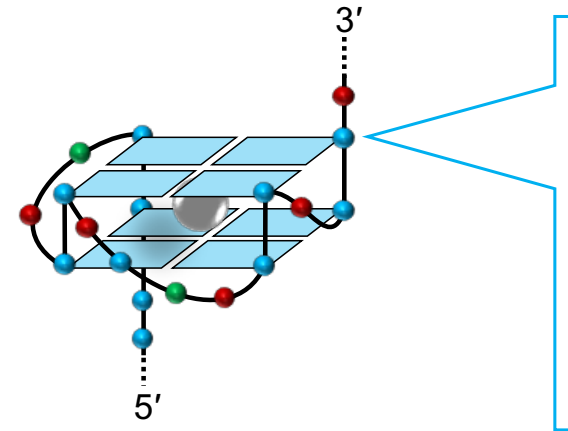

G-quadruplex structure

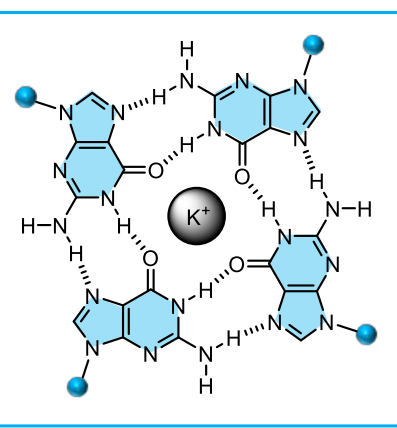

G-quartet

Figure 1. A) Top, EBNA1 sequence showing the central Gly-Ala repeat (GAr) domain surrounded by the $\mathrm{N}$ and $\mathrm{C}$ terminal domains; bottom, sequence of EBNA1 deleted for its GAr domain (EBNA1 $\mathrm{GAr}$ ) used in the biological assays described below. B) Left, the G-rich motif represented 13 times in the GAr-encoding sequence of EBNA1 mRNA; right, the putative parallel two-quartet RNA G-quadruplex proposed as a model.

Immune response to non-self proteins: The detection of exogenous proteins originating from virus, bacteria or parasites by the host immune system is achieved through the production of derived antigenic peptides by the proteasome and/or by abortive translation. The antigenic peptides are shuttled to the endoplasmic reticulum (ER) where they are assembled into protein complexes called the Major Histocompatibility Complex (MHC). The loaded MHC complexes are then transported to the surface of the host cell where they display the antigenic peptides to activate recognition by $T$ lymphocytes. Depending on the origin of the peptides (intra- vs. extracellular), the MHC are classified into class I and II, which are recognized by cytotoxic T-lymphocytes $\left(C D 8^{+} \mathrm{T}\right)$ and T-helper lymphocytes $\left(C D 4^{+} \mathrm{T}\right)$ respectively through specific membrane receptors. This process of antigenic peptides exposure, also called the antigen presentation pathway, allows the immune system to distinguish self from non-self, thereby ensuring the protection from viral, bacterial and parasite infections. ${ }^{11,12}$

EBNA1 and immune evasion: Like all the gamma-herpesviruses, EBV has evolved several strategies to evade the detection of its antigenic peptides and consequent elimination by the host immune system during both latent and lytic phases. Due to its crucial role in EBV genome replication and maintenance, and also to the fact that it is highly antigenic, EBNA1 plays a central role in the virus' strategy to escape immune detection during the latent phase. Indeed, EBNA1 protein is expressed in all dividing EBVinfected cells and $C D 8^{+} T$ cells directed towards EBNA1 epitopes exist in all infected individuals. Despite this, the immune system fails to detect and destroy EBNA1-expressing cells. To achieve this immune evasion, EBV has seemingly evolved a mechanism limiting EBNA1 production to the minimal level required for replication of the viral genome, but below the threshold for host immune detection. This 
is due to a tight regulation of the steady-state level of EBNA1 that minimizes the generation and, hence, the presentation of antigenic peptides. The immune evasion of EBNA1 depends on its central glycinealanine repeat $(\mathrm{GAr})^{13}$ and is based on the ability of EBNA1 to self-limit its own translation in cis (Figure 1, A). ${ }^{14-16}$ GAr consists of single alanine residues separated by 1-3 glycine residues, and is indeed a potent inhibitor of its own mRNA translation. ${ }^{17}$ The critical role of this domain was demonstrated through infection by an EBV strain encoding a GAr-deleted version of EBNA1 (EBNA1 $\triangle$ GAr), which induced high level of EBNA1 and efficient T cell response (Figure $1, A) \cdot{ }^{13}$ In addition, the ability of GAr to inhibit both its translation and antigenic presentation was shown to be tightly dependent on its length: longer GAr display a stronger inhibitory effect both on EBNA1 translation and antigen presentation. ${ }^{18}$ Another remarkable fact arguing for the strict involvement of the GAr domain is that the fusion of GAr upstream to any open reading frame causes suppression of both protein synthesis and antigen presentation; this feature was subsequently used for the design of a test model involving the expression of chicken ovalbumin (OVA/Gar-OVA, see below). A number of mechanisms have been proposed to explain the ability of GAr to self-control EBNA1 synthesis (and thus of EBNA1-derived antigenic peptides), but two main hypotheses are currently admitted: the first one is based on the role of EBNA1 mRNA structure (e.g., G-quadruplexes) and is the topic of this book chapter, whereas the second one is based on the role of EBNA1 protein itself. Importantly, these two mechanisms are not mutually exclusive, and one may envision that both are required for efficient immune evasion of EBV.

\section{G-Quadruplexes in the GAr-encoding sequence of EBNA1 mRNA}

Supporting the role of EBNA1 mRNA structure, it was found that the structure of the GAr-encoding sequence of EBNA1 mRNA, rather than the peptide sequence itself, was involved in the self-control of EBNA1 synthesis. ${ }^{19,20}$ Indeed, EBNA1 mRNA, in particular the sequence encoding GAr, is G-rich and form G-quadruplex (G4) structures. ${ }^{21}$ More recently, in 2014, Murat et al. identified a putative quadruplex-forming sequence (PQS) motif comprised of 18 nucleotides (encoding for Gly-Ala-Gly-AlaGly-Gly) and repeated 13 times in the full length 300-nucleotide-long GAr-encoding sequence of EBNA1 mRNA. ${ }^{22}$ A panel of optical spectroscopy (TDS, CD and UV-melting) and NMR measurements indicated that this short 18-nucleotide sequence forms a parallel G4 structure of high thermodynamic stability $\left(T_{\mathrm{m}}=54-56^{\circ} \mathrm{C}\right)$, likely corresponding to a two-quartet fold proposed as a model (Figure 1, B). Moreover, the 300-nucleotide long fragment also showed a CD signature typical of parallel G4 with a clear $\mathrm{K}^{+}$ dependency, convincingly suggesting that GAr-encoding mRNA may fold into multiple G4 units in a pearl-on-a-necklace manner, as observed for other repeated sequences like telomeres and minisatellites. ${ }^{23-25}$ It is straightforward to assume that G4 structures, like most secondary structures, have a higher probability of occurrence in long tandem repeat sequences, which can give rise to G4 clusters. However, although the two-quartet quadruplex model is likely to be the predominant conformation in vitro, a strong G4-polymorphism can be expected in vivo considering the very high Grich content of the GAr-encoding sequence of EBNA1 mRNA and the possible association of distal Gruns, which might be favored by formation of central long loops. ${ }^{26}$ Finally, although the thermodynamic and kinetic parameters that regulate G4 formation in vitro are well characterized, the conditions of their formation and the factors influencing their lifetime in vivo are more difficult to determine since many cellular factors, in particular nucleic acid-interacting proteins, may have a strong impact in a way or another. Thus, the number of G4 structures able to form in long tandem repeats in vivo clearly remains an open question. 
As stated above, a polymorphism exists with respect to the length of GAr domain, a fact which may reflect a way for EBV to adapt to its host. In addition, as previously mentioned, the translation inhibition of EBNA1 synthesis and presentation of the antigenic peptides are strongly dependent on GAr length, as shown both in mammalian cells and the budding yeast Saccharomyces cerevisiae. ${ }^{27}$ Hence, as the GAr length determines the number of PQS, it is tempting to correlate this structural feature with the biological response, which provides additional support to the G4-mediated regulation of EBNA1 expression.

The study of Murat et al. reported the inhibitory role of G4 structures on the translation of EBNA1 mRNA in vitro, suggesting a ribosome stalling mechanism. ${ }^{22}$ The authors also showed data that suggest that modulation of G4-RNA stability directly impacts EBNA1 levels, as antisense oligonucleotides that destabilize G4 (or prevent its formation) enhanced EBNA1 expression, whereas the G4-stabilizing compound Pyridostatin (PDS) decreases it (Figure 2). Following PDS treatment, inhibition of EBNA1 antigenic presentation was observed; at the same time, the authors did not exclude the involvement of other mechanisms due, in part, to the broad-spectrum activity profile of the drug. ${ }^{28}$ Also, it is important to mention that the method employed in this study to evaluate the effect of G4 ligands on EBNA1 expression is an in vitro coupled transcription-translation assay, which does not allow to distinguish between transcriptional and translational effects. This point is particularly relevant given that G4 ligands are also known to affect transcription, as for example the transcription of the human immunodeficiency virus (HIV) genome. ${ }^{29}$ Nonetheless, these observations are consistent with the repressor role of $\mathrm{G} 4$ observed in the translation of $5^{\prime}-\mathrm{UTR}^{30}$ and other inhibitory functions of mRNA processing caused by steric blockade effect of $\mathrm{G} 4 .{ }^{31,32}$
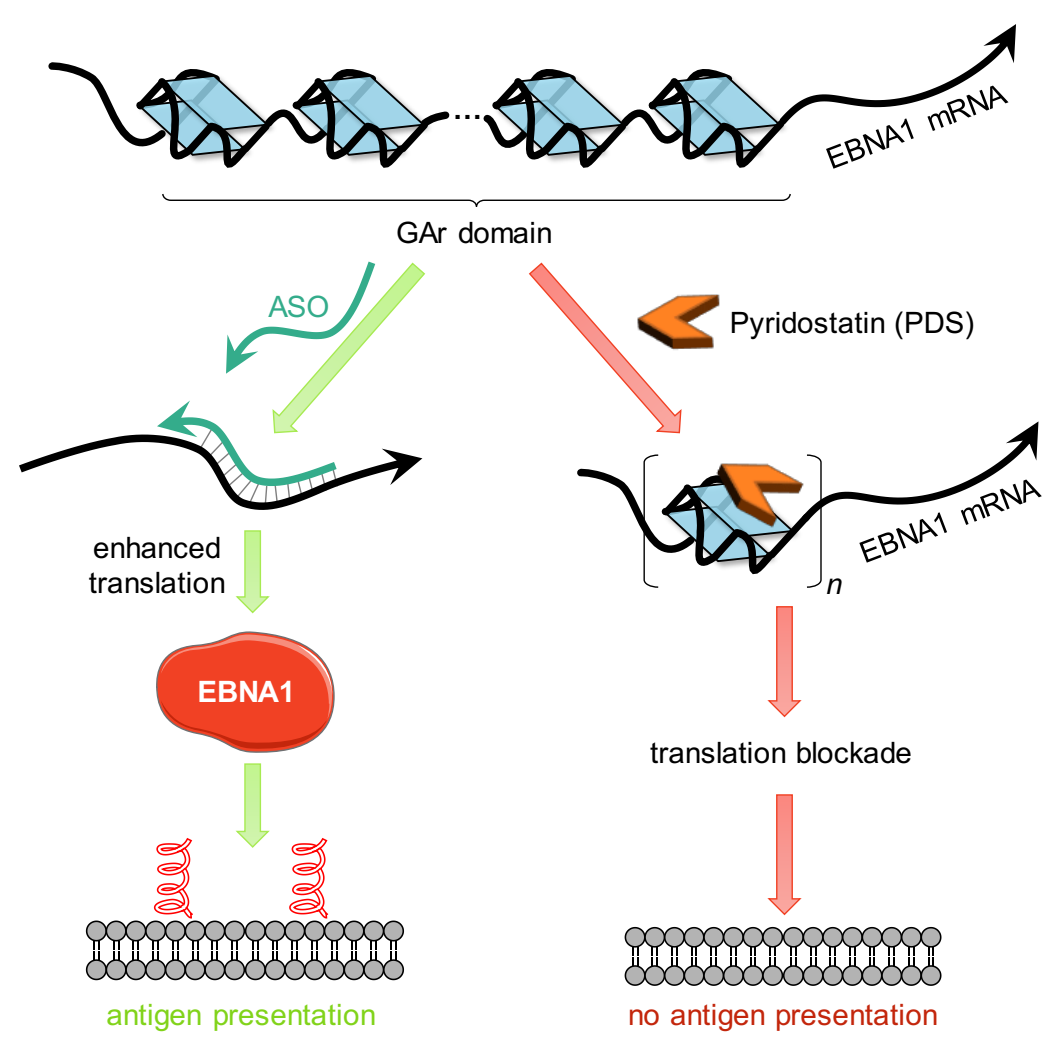

Figure 2. Schematic representation of a possible strategy of G4-mediated modulation of EBNA1 expression, as proposed by Murat et al. ${ }^{22}$ ASO = antisense oligonucleotide. 
In parallel, other mechanisms related to the involvement of G4 structures on self-inhibition of EBNA1 translation were considered. As mentioned above, EBNA1 protein itself was demonstrated to bind to G-quadruplexes in its own mRNA transcript with moderate affinity, a fact that was proposed to explain its self-regulation in cis. ${ }^{10}$ More recently, the synthesis of EBNA1 and its antigenic presentation was shown to be regulated by the host factor nucleolin (NCL). ${ }^{33,34}$ Altogether, these two studies set the stage to explore more in depth the interplay between G-quadruplexes that form in EBNA1 mRNA, NCL and self-regulation of EBNA1 synthesis.

\section{Nucleolin: a new player in the hide-and-seek game}

The role of NCL in the regulation of EBNA1 synthesis was uncovered thanks to a yeast (Saccharomyces cerevisiae)-based assay that recapitulates all the aspects of the GAr-based inhibition of translation including the GAr-length dependency. ${ }^{35}$ This assay was used to perform a genetic that aimed at identifying genes whose overexpression interfere (positively or negatively) with GAr-mediated translation inhibition. ${ }^{27,34}$ This way, the NSR1 gene which encodes the yeast homologue of human nucleolin (Nsr1p) was identified as able, when overexpressed, to exacerbate the inhibitory effect of GAr on translation, whereas its elimination (by knock-out of the NSR1 gene which is not essential in yeast)..$^{33}$ Follow-up investigations in yeast and in EBV-infected human cells confirmed and extend this effect to human nucleolin (NCL) and have shown that nucleolin is a conserved host cell factor critically involved in the GAr-based regulation of EBNA1 expression, a mechanism at the basis of EBV immune evasion. In particular, unambiguous demonstration was provided by overexpressing or downregulating NCL in three EBV-infected B-cells (Mutu-1, B95-8 and Raji), which led, respectively, to a decrease or an increase in EBNA1 expression, and to an increase of GAr-limited antigen presentation in the case of downregulation of NCL (Figure 3A)..$^{33}$ The inhibitory effect of NCL on EBNA1 synthesis was also studied in mammalian cells (HT1299 lung carcinoma cells) transfected with expression vectors encoding EBNA1 or its GAr-deleted version (EBNA1 $\triangle G A r$ ). As shown in Figure 3B (left), NCL downregulation induced EBNA1 repression only when the GAr domain was present, as no impact was observed on EBNA1 $\triangle \mathrm{GAr}$ protein. The same results were obtained with a second set of constructs encoding the chicken ovalbumin fused (or not) to a 235 amino acid-long GAr domain (235GAr-OVA vs. OVA), leading to the same conclusions (Figure $3 \mathrm{~B}$, right). These two settings represent benchmark assays giving crucial mechanistic information on the GAr-dependency of translation of EBNA1 or OVA, the latter further illustrating the generality of this mechanism of translation regulation. Importantly, the OVA/235 OVA assay also allows to determine the effect of GAr on antigen presentation on the host cell membrane using specific antibodies against SINFEKL (an OVA-derived antigenic peptide) associated to MHC class I receptors (Kb). Indeed, when fused to OVA, 235GAr lead to a strong inhibition of presentation of OVAderived antigenic peptide. ${ }^{16,35}$ Using this assay, NCL downregulation was shown to induce a significant GAr-dependent increase of antigen presentation (Figure 3C, left panel). ${ }^{33}$ Altogether, the test workflow described on Figure 3 have proven particularly useful for evaluation of GAr-interacting drugs and was used throughout our follow-up studies (below). 
A

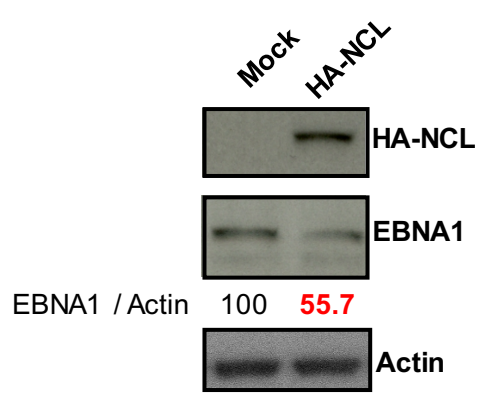

B
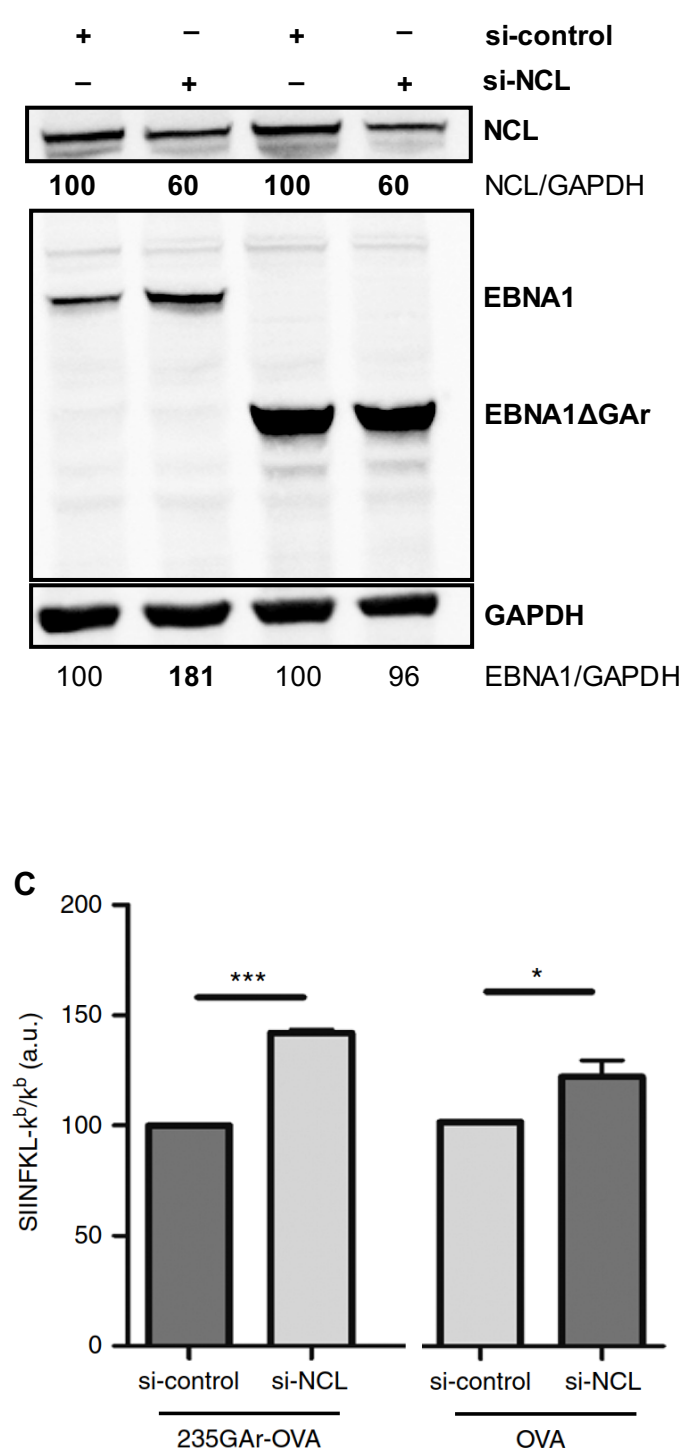

\section{HT1299 cells}

Mutu- 1 cells

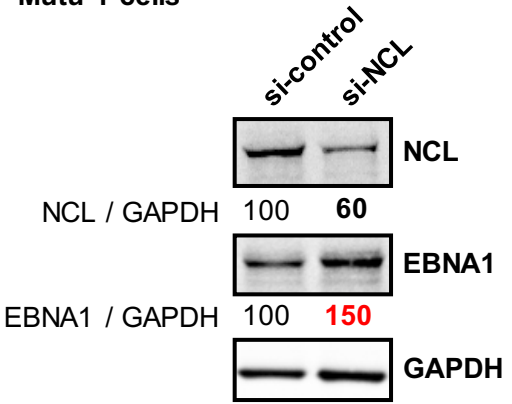

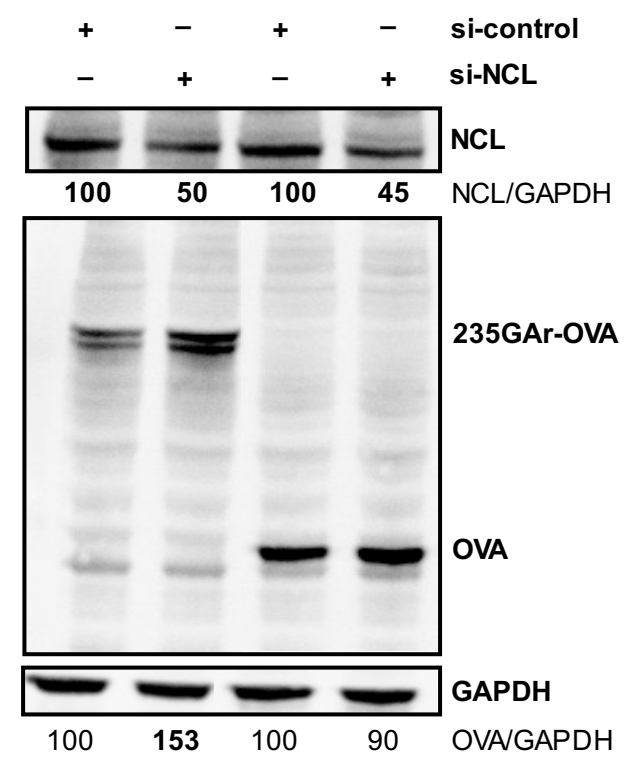
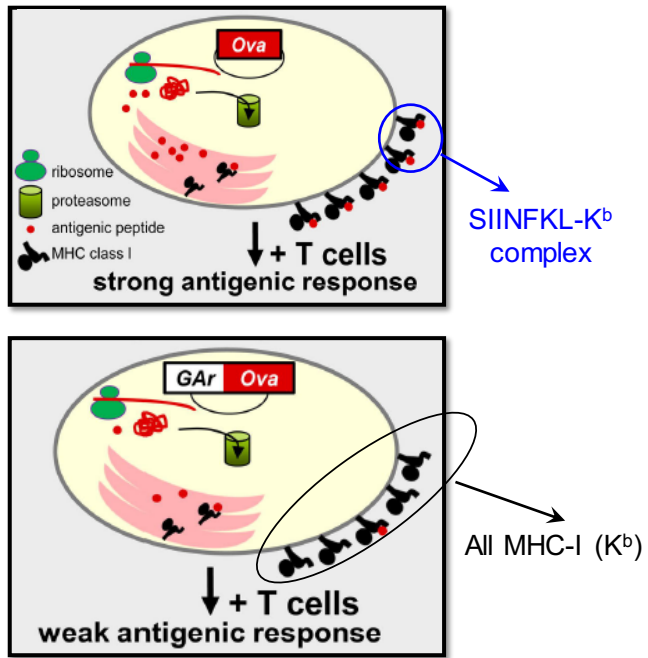

Figure 3. A) Western blot analysis showing EBNA1 expression level as a function of NCL overexpression (HA-NCL) (left) and downregulation (using siRNA, right) in EBV-infected Mutu-1 cells. B) Effect of NCL downregulation (using siRNA) on EBNA1 or EBNA1 $\mathrm{GAr}$ (left), or OVA or 235GAr-OVA (right) protein expression following transfection of HT1299 cells by the corresponding constructs. C) Effect of NCL downregulation (using siRNA) on antigen presentation after transfection of HT1299 cells with OVA or 235GAr-OVA and murine MHC class I K ${ }^{\text {b }}$ encoding plasmids. Cells were incubated with naive OVA $C D 8^{+} \mathrm{T}$ cells whose activation is measured. Adapted with permission form Ref. ${ }^{33}$. 
NCL is a multifunctional DNA/RNA-binding protein widely conserved among eukaryotes and implicated in many cellular functions such as ribosome biogenesis, rRNA maturation, cell cycle control, apoptosis, transcription and translation regulation. ${ }^{36}$ As such, $\mathrm{NCL}$ binds a variety of nucleic acids thereby showing a broad substrate promiscuity. NCL is one of the first G4-interacting protein identified. ${ }^{37}$ One of its best documented mechanism of action on $\mathrm{G} 4$ is its role as repressor of transcription through stabilization of G4 in the c-Myc promoter. ${ }^{38,39}$ More recently, NCL was shown to recognize G4-DNA within the LTR promoter of HIV, thereby silencing the provirus transcription. ${ }^{29} \mathrm{NCL}$ is ubiquitous and is therefore likely to interact at many G4 loci of DNA or RNA. The structural determinants of G4/NCL interactions are not really known, but recently three independent groups, including some of us, reported on the strong preference of $\mathrm{NCL}$ for binding G4-DNA harboring a long central loop. ${ }^{40-42}$ Whether this trend is a general rule that can be extended to recognition of G4-RNA remains to be explored. Therefore, after having uncovered the role of NCL in EBNA1 translation, our first line of investigation has been focused on finding whether NCL was directly interacting with the G4 formed in the GAr-encoding mRNA. This has been done by a classical affinity pull-down approach using the 18-nt oligonucleotide corresponding to the most probable G4-forming motif in the GAr-encoding sequence of EBNA1 mRNA (cf. Figure 1, A). This oligonucleotide sequence (g4-EBNA1), as well as a control mutated oligonucleotide containing adenine or uridine in place of guanine residues to prevent $\mathrm{G} 4$ folding (GM, negative control), and a 30nt RNA sequence known to bind NCL (ARPC2, positive control) were 3'-tagged by biotin, immobilized on streptavidin-covered sepharose beads, and incubated with lysates from HT1299 (lung cancer) cells, then thoroughly washed and eluted with increasing concentration of $\mathrm{KCl}(200-800 \mathrm{mM}$ ) (Figure 4, A$B)$. After elution, the pulled-down proteins were analyzed by western blotting against NCL. As shown from Figure 4, C, NCL was pulled down by g4-EBNA1 and RNA-G4 control (ARPC2), whilst no interaction was detected with the mutated sequence (GM). Importantly, the same results were obtained with recombinant NCL, showing that the interaction between NCL and G4 of the GAr-encoding sequence of EBNA1 mRNA is direct. ${ }^{33}$

A

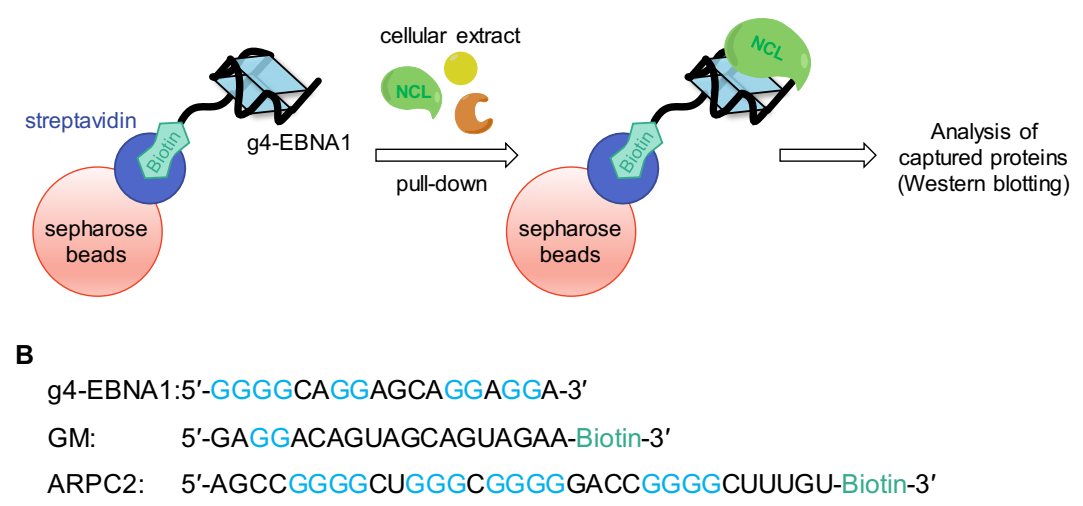

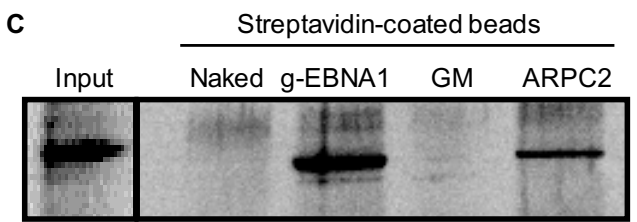

Figure 4. A) Schematic representation of the pull-down assay using G4-RNA immobilized on streptavidin-covered sepharose beads. B) RNA sequences used in the assay. C) Western blotting analysis of proteins after the pulldown. 
Affinity pull-down is a useful method for identification of proteins interacting with a given target (nucleic acid or protein), but should be considered only as a first indication. Therefore, other techniques are required to evaluate if the identified partners do indeed interact in a native environment. Thus, to verify whether the NCL-mEBNA1-G4 interaction was occurring in cellulo, a proximity ligation assay (PLA) was devised to detect this association in EBV-infected cells.

Briefly, PLA is a technique originally developed to detect proteins localized in close proximity to eachother (theoretically, at a maximum distance of $40 \mathrm{~nm}$ ) and is based on the use of a pair of antibodies raised in two different species, each targeting one of the two proteins of interest. By using oligonucleotides labelled with haptens (digoxigenin or biotin), PLA has been adapted to the study of protein-DNA and protein-RNA interactions. ${ }^{43}$ To this end, the RNA target (herein, EBNA1 mRNA) was tagged through in situ hybridization with a digoxigenin-labelled DNA probe detected by a mouse antidigoxigenin antibody, whereas NCL was recognized by a rabbit anti-nucleolin antibody. If the two partners are located in close proximity (strongly suggesting that they are interacting), subsequent incubation with secondary antibodies directed against each primary species and covalently coupled to specific DNA primers will generate a circular DNA template. The latter is then amplified by rolling circle amplification (RCA) and detected by in situ hybridization with specific fluorescent probes. Thus, the close proximity of the two partners is visualized through formation of bright fluorescent foci (PLA dots) that appear at the place of the monitored interaction. The principle of the PLA assay is depicted in Figure 5.

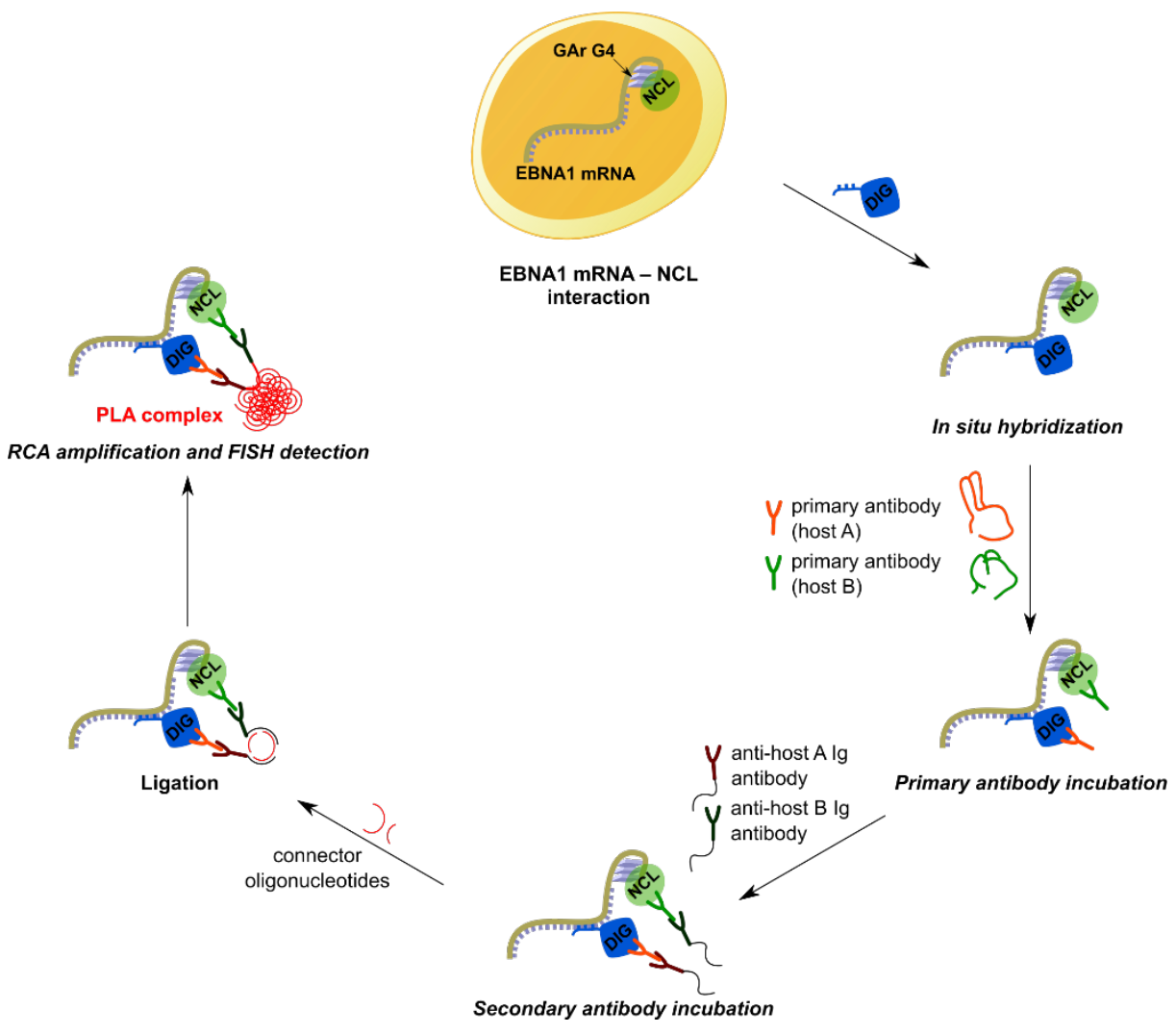

Figure 5. Principle of the proximity ligation assay (PLA) applied to the study of EBNA1 mRNA-NCL interaction. The DNA probe (5'-CTTTCCAAACCACCCTCCTTTTTTGCGCCTGCCTCCATCAAAAA-digoxigenin-3') was chosen such that hybridization occurs outside the G4-forming region, to avoid eventual interference between $\mathrm{G} 4$ formation and RNA-DNA hybridization. 
In a first step, to verify the specificity of the EBNA1-digoxigenin probe and the detection of the hybridization complex by anti-digoxigenin antibody, HT1299 (lung carcinoma) cells were transfected with an EBNA1 construct and analyzed by RNA in situ hybridization coupled to immunofluorescence (rISH-IF). Primary EBNA1-immunocomplexes were detected using an anti-mouse secondary antibody labelled with Alexa Fluor 568. Fluorescence imaging revealed the accumulation of EBNA1 mRNA in the nucleus (Figure $6 \mathrm{~A}$, red staining). In parallel, immunostaining was performed to determine the appropriate conditions for detecting endogenous NCL using a rabbit anti-NCL antibody (Figure $6 \mathrm{~B}$ ). Altogether, these assays demonstrated an accumulation of NCL and EBNA1 mRNA in the nucleus. Then the complete set of PLA reagents (i.e., EBNA1-rISH probe, primary antibodies, secondary antibodies, and RCA kit) was applied to the same EBNA1-transfected cell line (HT1299) and to an EBV-infected cell line (B95-8, EBV-producing marmoset lymphoblastoid cells). In both cell lines PLA complexes (depicted as white dots) were detected in or at close vicinity of the nuclear compartment (Figure $6 \mathrm{C}, \mathrm{D}$ ). This confirms that NCL binds to EBNA1 mRNA, and that this interaction mostly takes place in the nucleus of EBV-infected as well as EBNA1-transfected cells.
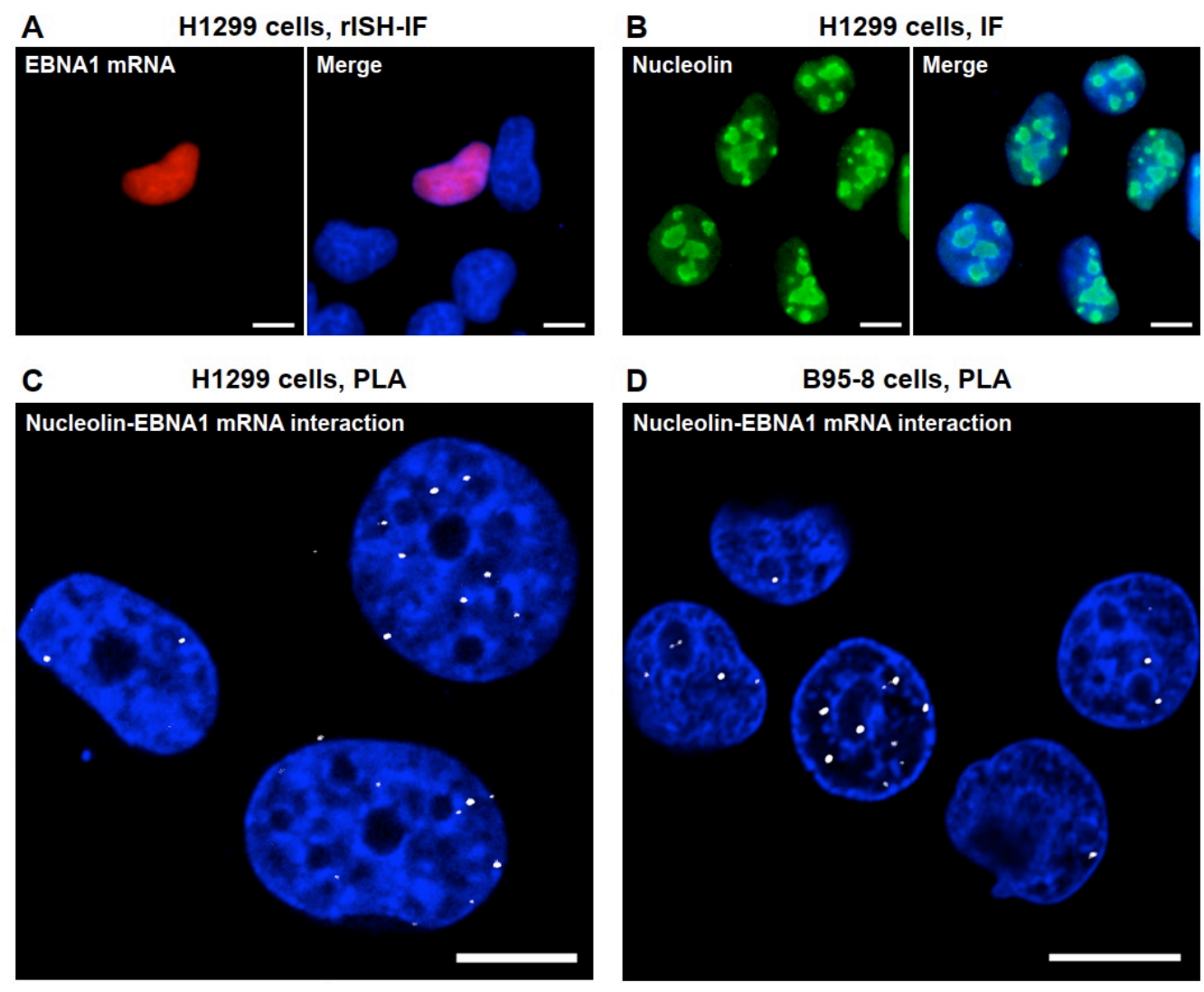

Figure 6. A) RNA in situ hybridization ( $\mathrm{r} / \mathrm{SH}$ ) with the EBNA1 digoxigenin probe followed by mouse antidigoxigenin immunostaining and anti-mouse IgG labelled with Alexa Fluor 568. DAPI was used for nuclear counterstaining. B) Detection of NCL by rabbit anti-NCL antibodies. C-D) PLA in C) EBNA1-transfected HT1299 cells and D) EBV-infected B95-8 cells. PLA was performed using mouse anti-digoxigenin and rabbit anti-nucleolin, followed by anti-rabbit and anti-mouse Ig PLA probes applied together with fluorescent probes for RCA product detection. Scale bars represent $10 \mu \mathrm{m}$. Adapted with permission from Ref. ${ }^{44}$.

The presence of PLA dots in the nucleus is consistent with the predominant nuclear localization of NCL and more specifically with the recent notion that early translation events coupled to transcription occur in the nucleus. ${ }^{45,46}$ In line, recent findings by some of us have shown that translation of EBNA1 
mRNA as well as antigen presentation may be regulated by NCL in the nucleus. ${ }^{47}$ Altogether, these results denote that EBV may exploit host cell mRNA maturation process(es) in order to hamper translation and thus immune response. Finally, and importantly, it was shown that the presence of GAr-encoding sequence, that is, the G4-forming motif of EBNA1 mRNA, was absolutely required for its interaction with NCL, as no PLA signals were detected when HT1299 cells were transfected with an EBNA1 construct lacking the GAr domain (EBNA1 $\triangle G A r) .{ }^{33}$ In summary, the yeast assay combined with PLA have been instrumental for unravelling the interaction between NCL and EBNA1 mRNA-G4 on one hand, and for visualizing and localizing this interaction in cells on the other hand. Thus, NCL is the first identified host cell factor involved in EBNA1 stealthiness and, as shown previously, NCL expression is directly linked to the regulation of EBNA1 level. For these reasons, the interaction of NCL with G4s in EBNA1 mRNA immediately appeared as a pertinent target for pharmacological intervention with the aim of modulating EBNA1 translation and, in fine, triggering immune response against EBV-infected cancer cells.

\section{PhenDC3, a benchmark G4 ligand, disrupts NCL-EBNA1 mRNA interaction, enhances EBNA1 expression, and overcomes the immune evasion of EBV}

Following these results, the effect of G4-interacting compounds (G4-ligands) on the NCL-EBNA G4 interaction and immune evasion of EBV was evaluated. As a first step, the benchmark G4-ligand PhenDC3 was examined since this compound exhibits high affinity (with $K_{\mathrm{d}}$ in the nanomolar range) and selectivity for most $\mathrm{G} 4$ structures and has been validated as one of the best probe for targeting $\mathrm{G} 4$ DNA or RNA in cells through numerous studies. ${ }^{25,48-52} \mathrm{~A}$ remarkable advantage of PhenDC3 is its high permeation capacity with regard to live mammalian cell lines and yeast (S. cerevisiae). In parallel, we also evaluated Pyridostatin (PDS), another benchmark G4-ligand widely used for in-cell G4-studies, which was shown by Murat et al. to inhibit EBNA1 expression through stabilization of G4s in EBNA1 mRNA (cf. Figure 2). Both compounds strongly stabilize $\mathrm{G} 4$ and, as such, are expected to increase the lifetime of G4, thereby emphasizing their biological roles. The question whether small molecules can enhance or disrupt G4-protein interactions was much less investigated and is highly topical, raising questions about the precise mechanism of action of quadruplex-targeting drugs. ${ }^{42,53,54}$

Prior to cell-based assays, the capacity of the two ligands to interact with the G4 structure of EBNA1 mRNA was evaluated in vitro, using classical biophysical assays (G4-FID and G4-FRET-melting) (Figure 7). The low $D C_{50}$ value (i.e., high displacement activity) and high $\Delta T_{\mathrm{m}}$ (i.e., thermal stabilization) determined in both cases indicate that the two compounds bind to and strongly stabilize the g4-EBNA1 structure. Also, excellent selectivity for G4 vs. duplex DNA was evidenced by the absence of impact of the duplex competitor (ds26) on the variation in melting temperature induced by the ligands. Thus, both PhenDC3 and PDS can be considered as strong binders of g4-EBNA-G4 used as a model structure with a slight, but significant, advantage for PhenDC3 that displayed globally a higher performance. 

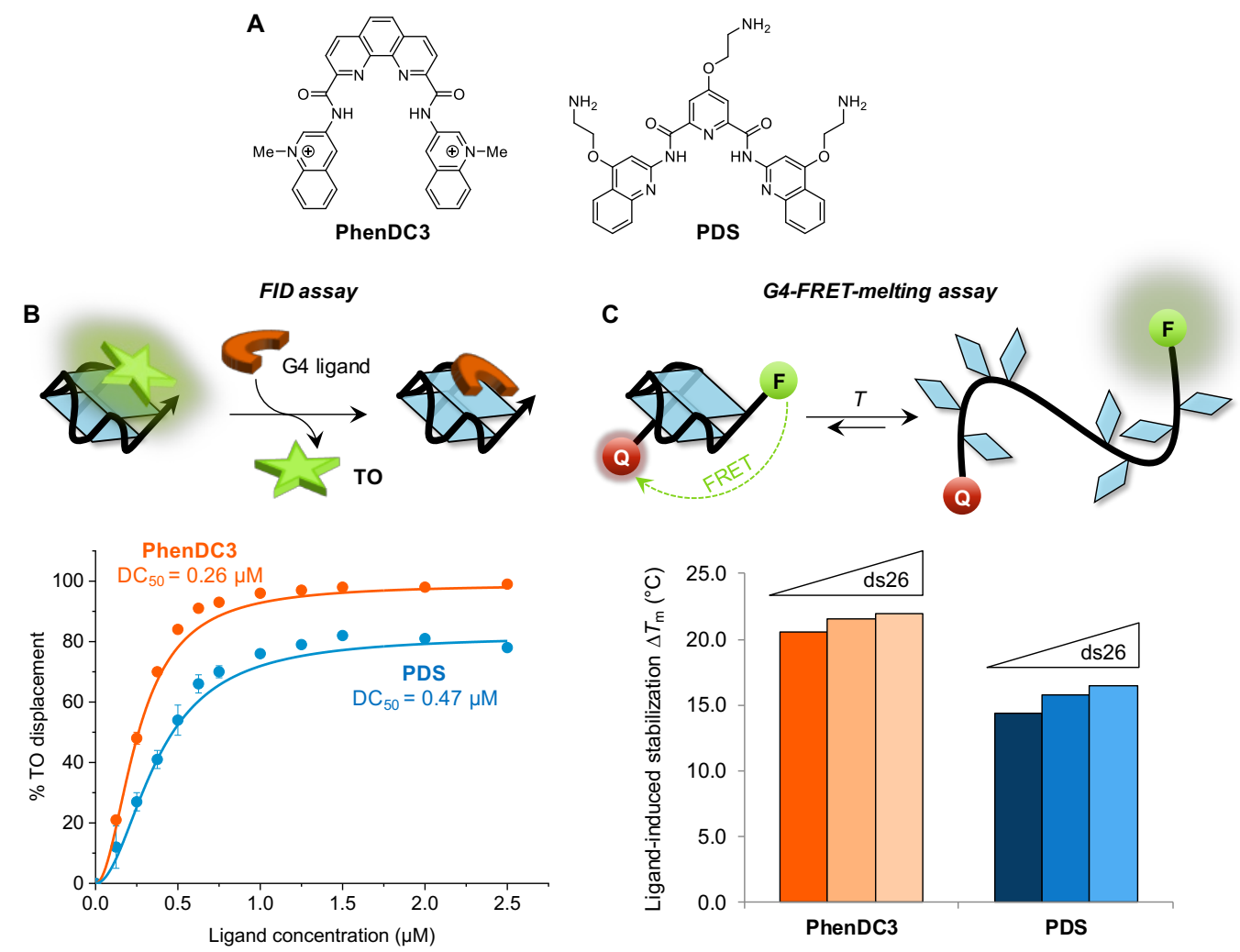

Figure 7. A) Chemical structures of PhenDC3 and Pyridostatin (PDS) and evaluation of their G4 binding properties to G4-RNA oligoribonucleotide (g4-EBNA1) in vitro by B) FID (fluorescent-indicator displacement) assay and C) FRET-melting assays. The principle of each assay is illustrated above the graphs. TO = Thiazole Ornage dye; $\mathrm{DC}_{50}$ $=$ ligand concentration inducing $50 \%$ decrease in TO fluorescence; $\Delta T_{\mathrm{m}}\left({ }^{\circ} \mathrm{C}\right)=$ increment of melting temperature induced by the ligand alone or in presence of a 26-bp duplex competitor (ds26).

We then took advantage of the previously developed PLA assay to examine the direct in cellulo effect of both compounds on the NCL-GAr interaction. To this end, B95-8 cells were treated with PhenDC3 and PDS at sub-toxic concentration $(<1 \mu \mathrm{M})$ to avoid cell death, then fixed and PLA was performed. A qualitative look at the resulting confocal fluorescence images indicates clearly that PhenDC3 reduces significantly the PLA signal as compared to the untreated control, whereas PDS does not impact it (Figure 8, A). PLA is most often used as a qualitative method to visualize and localize the interaction between two biological partners in cells. However, in the case of drugs acting on a given interaction, quantitative evaluation is absolutely required to draw proper comparison of drug activity within a series. Thus, a protocol was further developed to achieve quantitative PLA. In detail, one hundred cells from control (DMSO) and drug-treated groups were imaged and the number of interactions (i.e. the number of PLA dots) per cell was counted using a customized image analysis protocol as depicted in Figure $8, B .{ }^{44}$ The experiments were done in triplicate to obtain accurate quantification. As shown from the resulting histograms (Figure 8,C), upon treatment with PhenDC3 the number of PLA dots per cell was reduced by a factor of 3.3 (from $3.6 \pm 0.3$ to $1.1 \pm 0.4$ ), whilst this number was not significantly modified in PDS-treated cells $(3.7 \pm 0.9)$. 


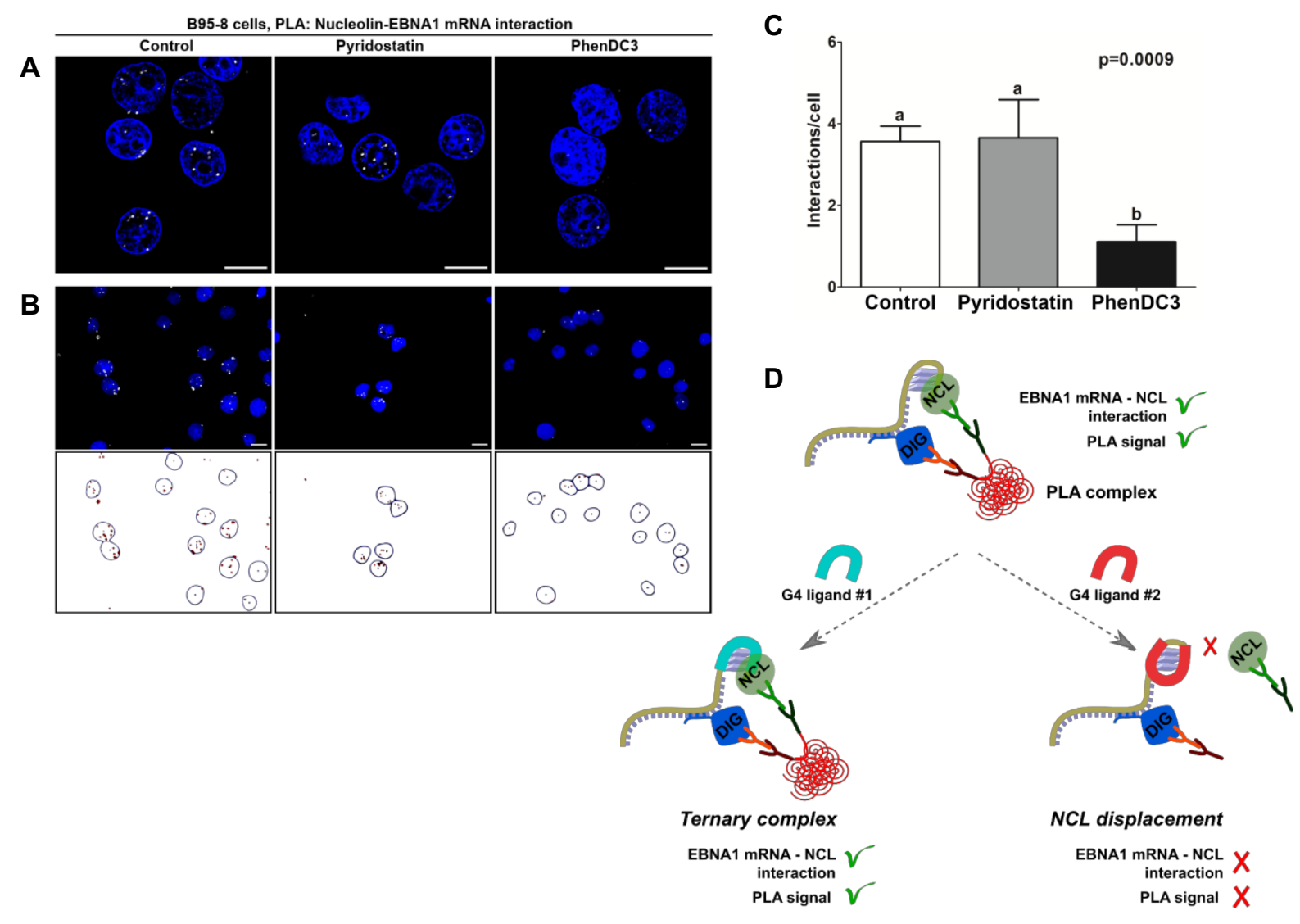

Figure 8. Evaluation of PhenDC3 and PDS in B95-8 cells using the quantitative PLA assay. A) Microscopy images of cells treated with DMSO (control), PhenDC3 or PDS (5 $\mu \mathrm{M})$. Nuclei are stained by DAPI (blue). White dots (PLA signals) indicate the interaction between NCL and EBNA1 mRNA. B) Quantification of PLA signals: 100 cells per condition were imaged and the number of dots per cell was estimated using a customized protocol in ImageJ. C) Graph showing number of PLA dots per cell as function of ligand. D) Schematic representation of the proposed mode of action of G4-ligands explaining the different effects on the PLA patterns. Left: Ternary interaction (G4RNA/NCL/ligand) induces no modification of the PLA dots as compared to control; right: competitive binding of ligand displaced NCL and reduces the number of PLA dots. Adapted with permission from Ref. ${ }^{44}$.

Thus, the quantitative PLA clearly indicates that PhenDC3 can disrupt the interaction between NCL and G4 that form in the GAr-encoding sequence of EBNA1 mRNA in a cellular context, whilst PDS has no effect. This discrepancy between the two G4 ligands can be explained in several ways. If we assume that NCL and drugs have common binding site(s) on GAr-G4, then competitive binding is possible and the result reflects the relative affinities of the endogenous (i.e., protein) and exogenous (i.e., small molecule) partners. In line, the higher affinity of PhenDC3 enables a competition with NCL for G4 binding and thus disrupts the interaction of NCL with GAr-mRNA. On the opposite PDS, that has a significantly lower G4 affinity cannot prevent NCL-mRNA association. Alternatively, it could be speculated that the drug (e.g., PhenDC3) induces a strong conformational change of the G4-mRNA target thereby hampering further recognition by NCL, while the non-active drug (PDS) does not modify the G4 structure. Finally, the drug may be bound to an allosteric site (G-quartet, groove, or loop) that does not disturb RNA recognition by the protein (Figure $8, D$ ). This case has been reported for carboxyPDS and 7ODT (hexaoxazole analogue of telomestatin) that were shown to bind G4 without affecting their recognition by the antibody or proteins, namely through formation of ternary complexes. ${ }^{55,56}$ Finally, since the drugs are incubated in living cells, differences in cell uptake, or 
subcellular distribution, or metabolism cannot be excluded, and may contribute to various extents to the observed differences in activity.

To further explore the comparison between PhenDC3 and PDS with regard to NCL/GAr-G4 interaction, we used the affinity pull-down assay described in Figure 4. Beads coated with g4-EBNA1 were incubated with endogenous or recombinant NCL in presence or in the absence of the drugs. In good agreement with the PLA results, inhibition of NCL binding to beads was achieved in presence of PhenDC3, whereas, in the same conditions, PDS had no effect (Figure 9). The same trend was also observed with the positive control G4-RNA (ARPC2) that also binds NCL.

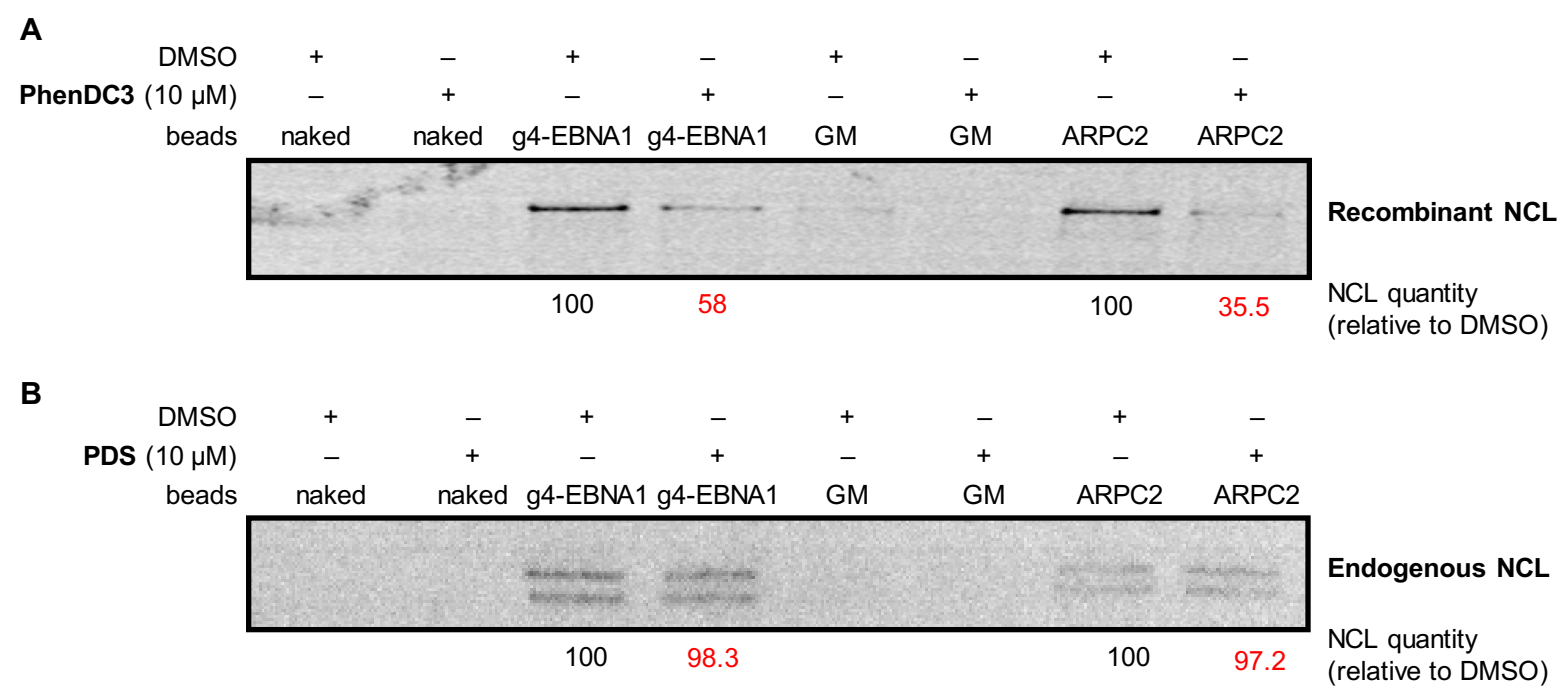

Figure 9. Inhibition NCL-G4 binding by PhenDC3 and PDS, as determined by the affinity pull-down assay. for the principle of the assay, cf. Figure 4, A. Adapted with permission from Ref. ${ }^{33}$.

Interestingly, we recently investigated in vitro the ability of G4 drugs to disrupt NCL interaction with G4-DNA and found again that PhenDC3 was the more efficient competitor of NCL, as compared to PDS and other G4-binding compounds (RHPS4, 360A) frequently used as G4 probes. ${ }^{42}$ Therefore, our data from PLA and pull-down assays are fully consistent with these recent findings and demonstrate that G4 ligands that exhibit comparable binding parameters for a given G4 structure in vitro may behave differently in cellulo, in part due to the differences in their capacity to compete with associated proteins.

In conclusion, it is worth noting that PLA coupled with quantitative imaging represents an attractive alternative to overcome the limitations of in vitro assays and to characterize the G4-RNA binding drugs targeting specific protein-RNA interactions in cells. In particular, this approach may represent a new and powerful tool for ranking and refining the selection of drug candidates during "hit-to-lead optimization" processes.

Biological effects: The effects of the two compounds on GAr-mediated protein expression inhibition and on antigenic stimulation have been evaluated using the set of biological assays described above. First, HT1299 cells transfected with plasmid expressing the ovalbumin fragments (OVA/235GAr-OVA) were treated, or not, with PhenDC3 or PDS ( $5 \mu \mathrm{M}, 48 \mathrm{~h}$ ) and OVA and 235GAr-OVA expression levels were evaluated by western blotting (Figure 10). This analysis revealed that PhenDC3 induced a 
significant increase of the steady state level of 235-OVA while producing no notable variation of OVA expression (Figure 10 A). This result demonstrates that PhenDC3 is able to affect the protein expression exclusively when the G4-forming domain (235GAr) is present in the construct. As shown from control experiments, the corresponding mRNA levels were not modified, allowing to conclude that, at least in these experiments, PhenDC3 enhances the expression of the protein without significant effect on mRNA level. As both EBNA1 and OVA are very stable proteins, PhenDC3 most probably increases EBNA1 translation. In stark contrast, no clear effect on 235GAr-OVA or OVA was observed after treatment with PDS in the same conditions (Figure 10A). The effect of the two drugs on the expression of viral EBNA1 was also evaluated in two EBV-infected cancer cell lines, Mutu-1 (Burkitt's lymphoma) and NCP-6661 (nasopharyngeal carcinoma). Western blotting analysis indicated that PhenDC3 treatment enhanced EBNA1 synthesis (Figure 10B). Again, PDS showed no activity even when applied at a higher concentration range as compared to PhenDC3 (up to $9 \mu \mathrm{M}$, Figure 10C). These data are fully consistent with the capacity of the compounds to disrupt (or not) the NCL-EBNA1 mRNA G4 interaction observed by PLA and in pull-down experiments and confirm that pharmacological modulation of NCL binding to G4 of EBNA1 mRNA is feasible, paving a way to counteract the inhibitory control of GAr on the EBNA1 production in EBV-infected cancer cells.
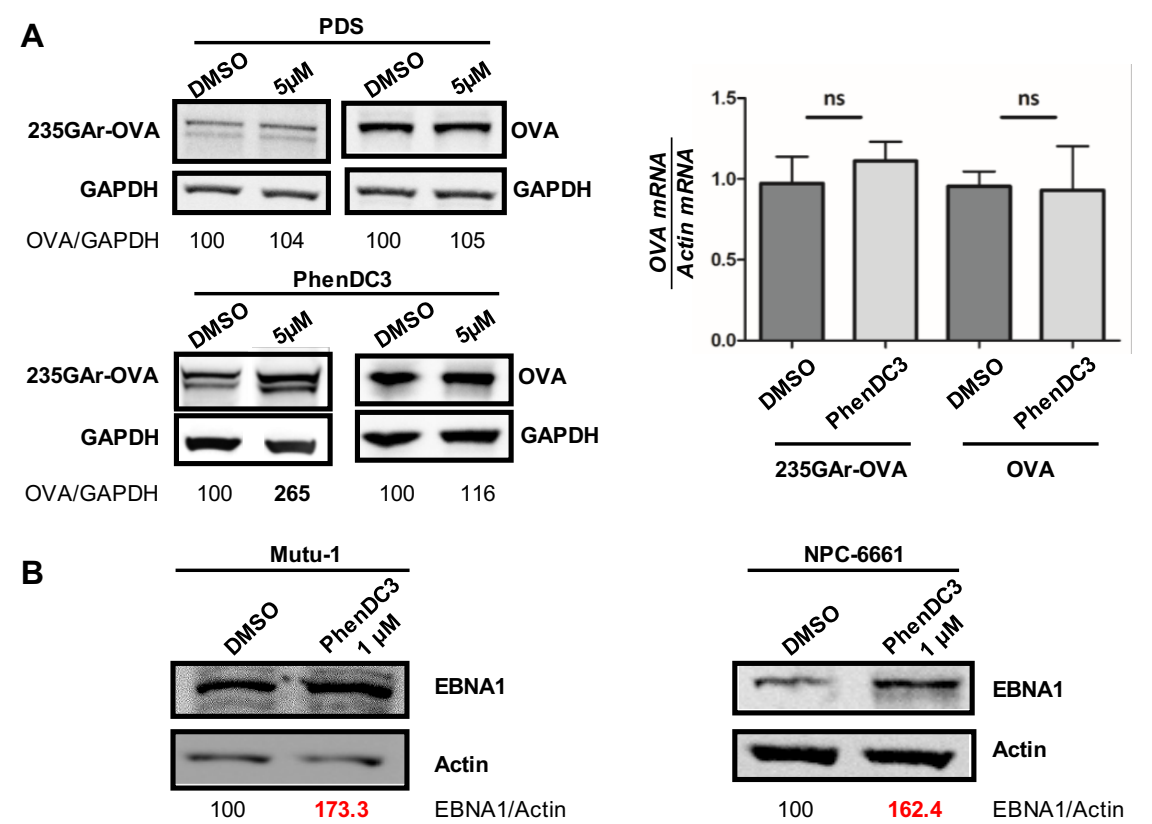

C
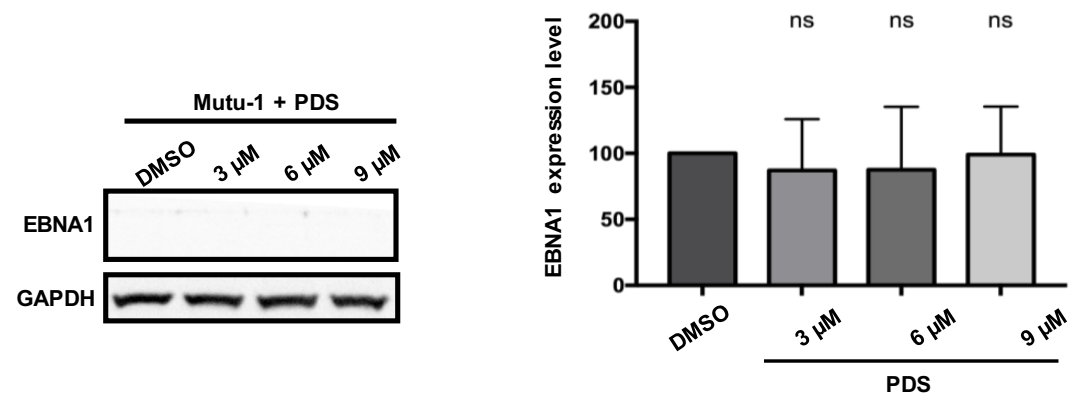

Figure 10. Protein expression after treatment of cells with PhenDC3 or PDS. A) HT1299 cell line transfected with OVA or 235GAr-OVA constructs, western blotting (left) and mRNA quantification (right). B) Western blotting analysis after treatment of Mutu-1 and NCP6661 with PhenDC3 $(1 \mu \mathrm{M})$. C) Western blotting analysis (left) and quantification of EBNA1 level (right) after treatment of Mutu-1 cells with PDS at concentrations of 3, 6 or $9 \mu \mathrm{M}$ (left). 
Finally, in contrast to the study of Murat et al., in our hands PDS showed no significant effect on the translation of EBNA1 despite its high binding affinity for EBNA1-G4 as determined by FID and FRETmelting. Obviously unfavorable pharmacological features (poor uptake, undesired subcellular distribution) and eventual off-target binding affecting PDS capacity to reach G4 in EBNA1 mRNA could explain this discrepancy. Of note, differences in experimental conditions between the two studies should also be considered: indeed, PDS was initially evaluated using an in vitro transcriptiontranslation coupled assay, thus it cannot be excluded that the impact on EBNA1 synthesis was, at least in part, due to effect of the compound on transcription rather than on translation. ${ }^{22}$

As a next step, PhenDC3 has been evaluated with respect to its ability to stimulate the presentation of antigenic peptide that, as shown before, is directly related to increase in EBNA1 level. This was done using the OT1 T-cell proliferation assay as described previously (Figure 11), again employing the HT1299 cells transfected with OVA or 235GAr-OVA constructs. As shown on Figure 11, PhenDC3 significantly increased (2-fold change) the proliferation of T cells added to the pool of cells expressing 235GAr-OVA, whereas it has no effect on OT1-T cells incubated with the OVA-expressing pool of cells. The stimulation of the immune response induced by PhenDC3 is fully consistent with the inhibitory role of NCL on antigen presentation and with the ability of PhenDC3 to interfere with the binding of NCL on the G4 motifs of EBNA1 mRNA. This data is in sharp contrast with the observations by Murat et al. who found that PDS further decreases GAr-restricted presentation of antigenic peptides.
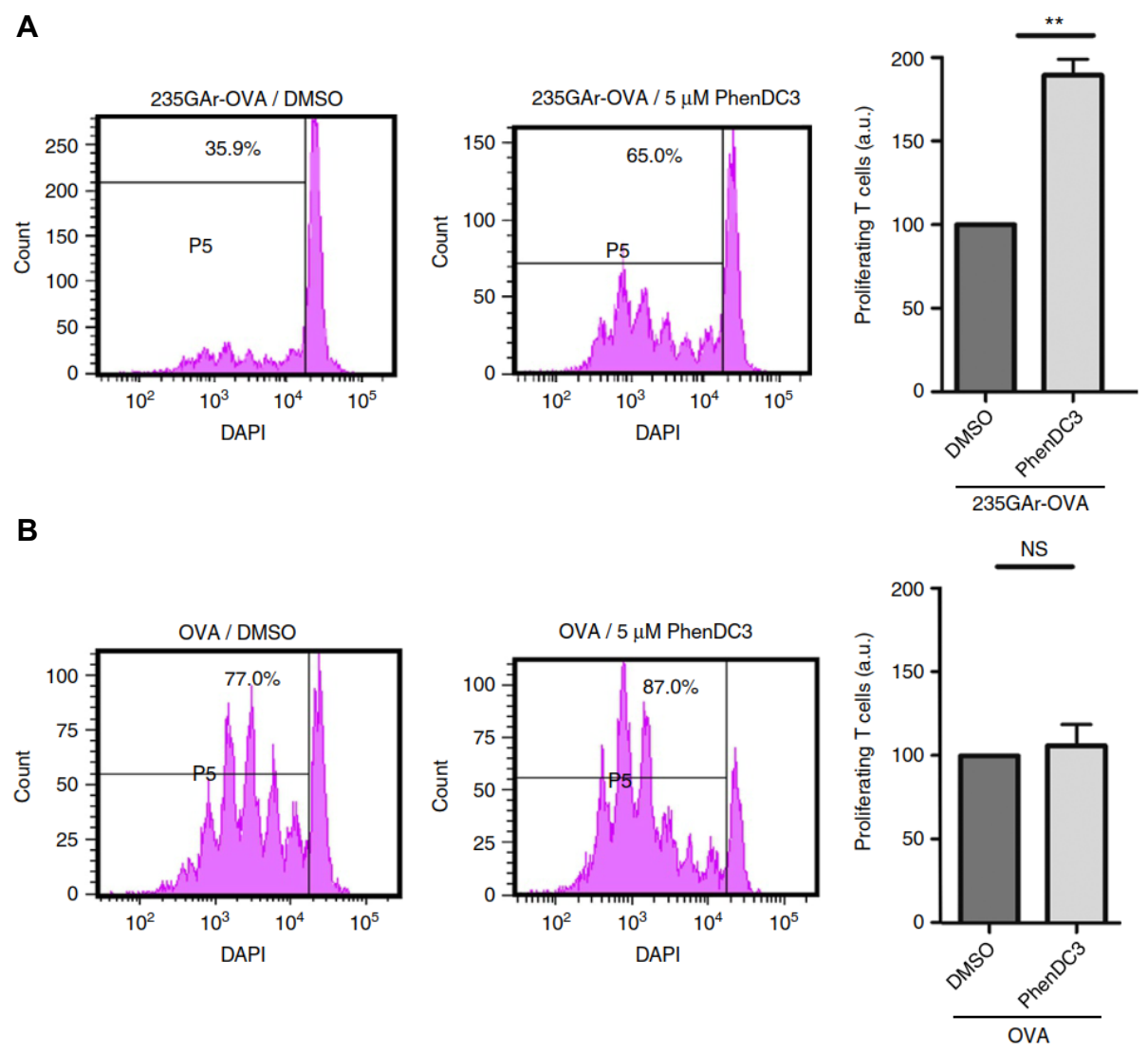

Figure 11. FACS analysis of T-cell proliferation (T-cell assay). HT1299 cells were transfected with mouse $\mathrm{K}^{\mathrm{b}}$ and $\mathrm{A}$ ) 235GAr-OVA or B) OVA plasmids, and treated with PhenDC3 $(5 \mu \mathrm{M})$ or DMSO (control). Cells were mixed with mouse naïve $\mathrm{OVA}_{257-264}$ (SINFEKL) specific $\mathrm{CD}^{+}$T-cells and stained with Celltrace Violet. Quantification of proliferating $\mathrm{T}$ lymphocytes by FACS following treatment are shown in the graphs on the right (ns = not significant). Reproduced with permission from Ref. ${ }^{33}$. 
Altogether, and despite these discrepancies, it can be concluded from these two studies that $\mathrm{G} 4$ motifs formed in the EBNA1 GAr domain are negative regulators of EBNA1 synthesis, acting either through $\mathrm{NCL}$ recruitment (our work) or as ribosome roadblocks (the work of Murat et al). Of note, these two possibilities are not mutually exclusive.

Taken together, our data demonstrate, firstly, that the role of NCL in GAr-based inhibition of translation involves its ability to bind to G4 in GAr-encoding sequence of EBNA1 mRNA. Secondly, this work provides a proof of concept that disrupting the NCL-G4 EBNA1 mRNA in cells by G4 ligands increases the GAr-restricted antigenic presentation of EBNA1, thus validating this class of compounds as potential agents to unveil EBV to the immune system and thereby for treating and/or preventing EBV-related cancers. However, in cellulo PLA imaging combined with in vitro biophysical data clearly indicate that the ability to disrupt in cellulo the NCL-G4 EBNA1 mRNA interaction is not a general feature shared by all G4 ligands. Further illustration of this specific point recently came from a novel series of G4 ligands (vide infra). Finally, from a practical viewpoint, this corpus of studies allowed to establish a pipeline of in vitro and in cellulo benchmark assays amenable to robust and drastic selection of drug candidates.

Following this work, we launched a program to identify other compounds with higher patentability and higher therapeutic index (or patentable properties). First, a selection of G4-selective and non-G4selective DNA ligands (Figure 12) was screened by western blot to assess their effect on EBNA1 synthesis in human cells. The results confirmed PhenDC3 as a "hit" dramatically enhancing EBNA1 production, as well as tBisQ-Cu and a number of other G4-binding metal complexes. ${ }^{57}$ At the same time, it was observed that other G4 binders belonging to different chemical classes, such as condensed polyaromatic compounds (i.e., MMQ1, MMQ12, TrisQ) and styryl dyes (PhenDV) either decreased, or had no effect on EBNA1 expression. Most interestingly, cationic bis(acylhydrazone) PyDH2 synthesized in our laboratory enhanced EBNA1 expression to a level comparable to the one induced by PhenDC3, as could be judged from this semi-quantitative screen. On these premises, a medicinal chemistry program was launched to devise a second generation of compounds derived from PhenDC3 and PyDH2 with optimized properties and evaluate a broader range of derivatives. 


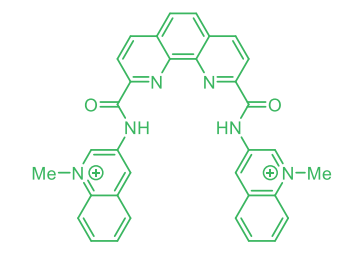

PhenDC3

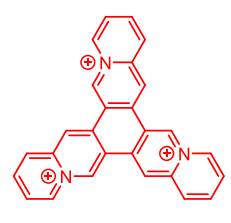

TrisQ

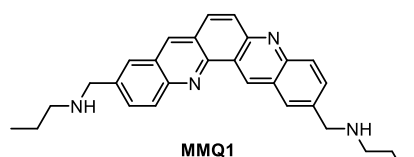

MMQ1

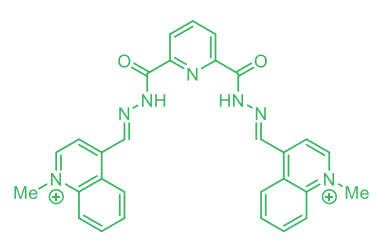

PyDH2

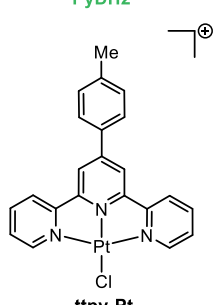

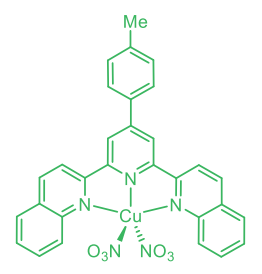

tBisQ-Cu

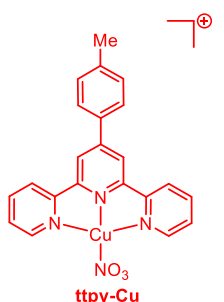

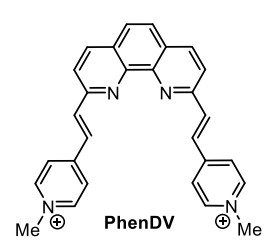
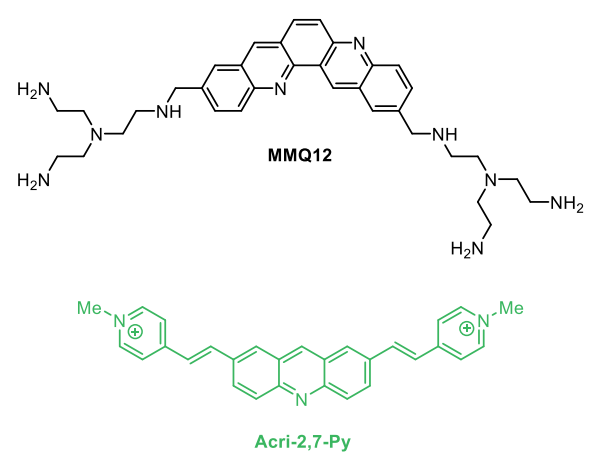

Figure 12. Small-molecule DNA and RNA binders tested as modulators of EBNA1 synthesis. Compounds that increased the expression of EBNA1 are colored in green; those that decreased EBNA1 expression are colored red; those shown in black had no significant effect. Acri-2,7-Py is a non-G4-selective DNA binder.

\section{Development of a novel series of G4-interacting ligands disrupting the NCL-G4 EBNA1 interaction}

With the aim to explore novel pharmaceutical scaffolds as putative G4 ligands and disruptors of the NCL-EBNA1 G4-mRNA interactions, a series of cationic bis(acylhydrazones) ("HetDH") was designed as shape analogues of PhenDC3. In addition, the $\mathrm{N}$-acylhydrazone group has been selected as a privileged scaffold in drug design, due to a combination of hydrogen-bond acceptor and donor sites capable of interactions with a wide range of biomolecules. Despite the fact that acylhydrazone derivatives are rapidly metabolized, this motif is encountered in several approved drugs (e.g., nifuroxazide and dantrolene $)^{58}$ and drug candidates. ${ }^{59-61}$ The facile synthetic availability of acylhydrazone derivatives, which allows furnishing congeneric series of compounds with distinct physico-chemical properties and bioactivities, makes it a promising scaffold in the drug discovery field. ${ }^{62-64}$

The design of the HetDH series features a central heterocyclic core (Het), i.e., a residue of pyridine (Py), pyrimidine (Pym), 1,8-naphthyridine (Naph), or 1,10-phenanthroline (Phen), connected via two acyhydrazone linkages to lateral, $\mathrm{N}$-substituted cationic heterocycles $(\mathrm{Ar}=$ pyridinium or quinolinium, $\mathrm{R}=\mathrm{Me}$, Et, or Bn). Altogether, we obtained 20 HetDH derivatives (PyDH1-5, PymDH1-5, NaphDH1-5, and PhenDH1-5, Table 1) in good yields by combinatorial synthesis starting from bis-acylhydrazides and cationic heteroaromatic aldehyde precursors. ${ }^{65}$ 
Table 2. Generic chemical structure and members of the HetDH family of bis(acylhydrazone) ligands.

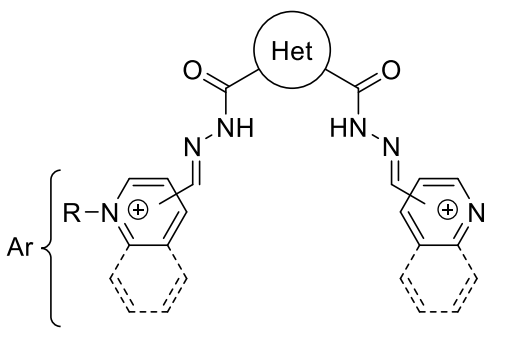

HetDHn

\begin{tabular}{|l|l|l|l|}
\hline \\
\hline Pym
\end{tabular}

In silico evaluation: The drug-like properties of HetDH derivatives were initially evaluated through the use of physicochemical descriptors, allowing the estimation of pharmacokinetics and drug-likeness of small molecules. ${ }^{66}$ The results of in silico assessment indicated satisfactory bioavailability for most HetDH derivatives, as given by the combination of six physicochemical descriptors (lipophilicity, molecular weight, polarity, solubility, saturation and number of rotatable bonds). However, insufficient saturation (i.e., low fraction of $s p^{3}$ carbons) could be identified as the limiting factor for bioavailability of most compounds, as well as high molecular weight $(M>700 \mathrm{Da})$ in several cases (NaphDH3, NaphDH5, PhenDH3 and PhenDH5. With the exception of these four compounds, all HetDH derivatives satisfied the Lipinski's rule, and most derivatives also satisfied the Muegge's rule, ${ }^{67}$ indicating a high potential to serve as drugs. Finally, gastrointestinal absorption prediction was made using the BOILED-egg model (Figure 13, A). ${ }^{68}$ According to this model, most compounds, again with the exception of NaphDH3, NaphDH5, and PhenDH3-5, could have a high rate of passive gastrointestinal absorption; however, none of compounds could cross the blood-brain barrier. In conclusion, the results of in silico evaluation indicate that HetDH scaffolds are compatible with their use as RNA-G4 
targeting drugs, while heavy and aromatic substitutes (e.g., $R=$ benzyl) should be avoided for the sake of bioavailability.
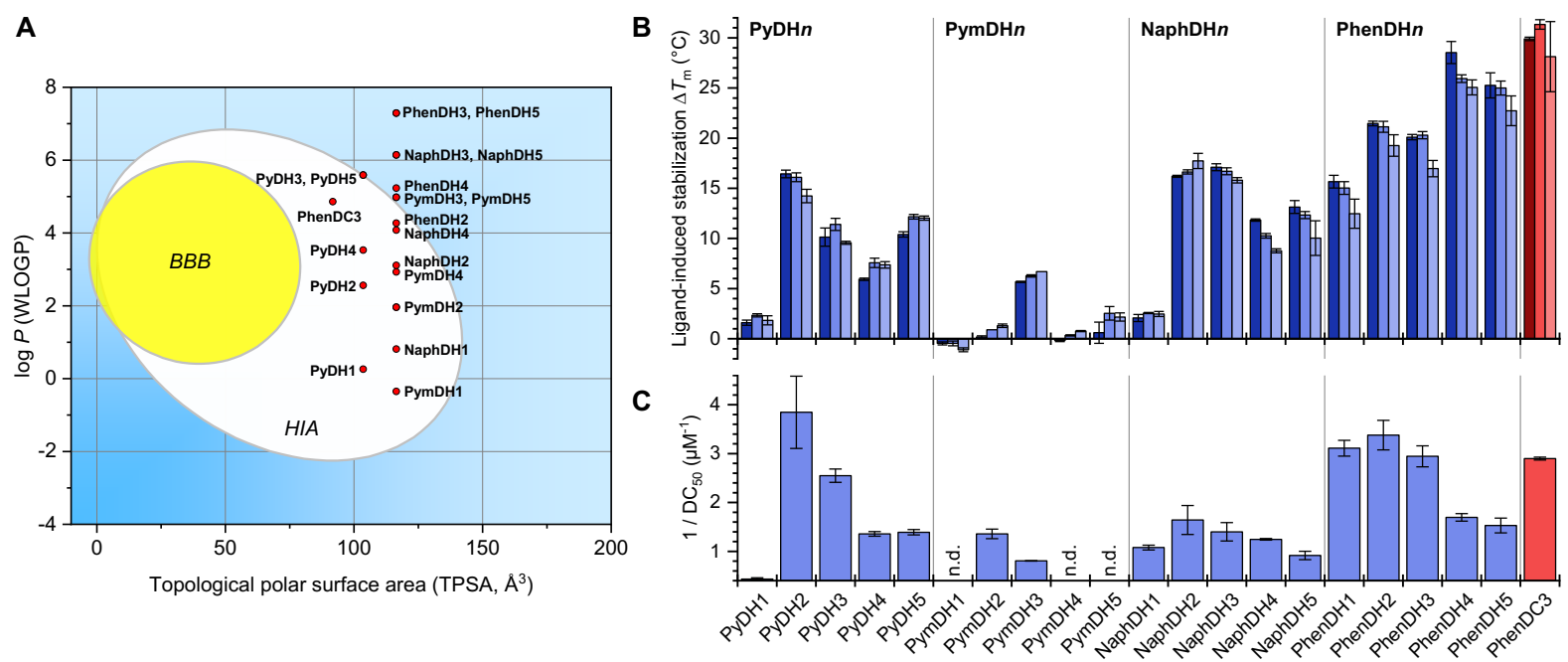

Figure 13. A) BOILED-egg evaluation ${ }^{68}$ of passive gastrointestinal absorption (HIA) and brain (BBB) penetration of HetDH derivatives. The white region indicates high probability of passive gastrointestinal absorption, while the yellow region indicates high probability of BBB penetration. B-C) In vitro assessment of binding of HetDH compounds and PhenDC3 (red) to g4-EBNA1: B) Thermal stabilization F-g4-EBNA1-T $(0.2 \mu \mathrm{M})$ by tested compounds $(1 \mu \mathrm{M})$, assessed by fluorescence melting experiments in the absence (dark blue bars) or in the presence of duplex DNA competitor ds 26 (blue bars: $3 \mu \mathrm{M}$, pale blue bars: $10 \mu \mathrm{M}$ ). Conditions: $10 \mathrm{mM} \mathrm{LiAsO} \mathrm{Me}_{2}$, $10 \mathrm{mM} \mathrm{KCl}, 90 \mathrm{mM} \mathrm{LiCl}$ buffer, pH 7.3. C) Ligand-induced displacement of TO $(0.5 \mu \mathrm{M})$ from g4-EBNA1 $(0.25 \mu \mathrm{M})$. n.d. = no displacement $\left(\mathrm{DC}_{50}>2.5 \mu \mathrm{M}\right)$. Conditions: $10 \mathrm{mM} \mathrm{LiAsO} \mathrm{Me}_{2}, 100 \mathrm{mM} \mathrm{KCl}, 1 \mathrm{mM} \mathrm{EDTA}$, buffer, $1 \% \mathrm{v} / \mathrm{v}$ DMSO. Adapted with permission from Ref. ${ }^{65}$.

Biophysical assessment: The capacity of HetDH derivatives to bind to g4-EBNA1 quadruplex was studied in vitro using the previously described biophysical methods, namely FRET-G4-melting and G4FID assay. Interestingly, these derivatives displayed great variability with respect to their capacity to bind to and stabilize the $\mathrm{G} 4$ target, revealing some structure-activity relationships. Specifically, most derivatives of the PyDH and NaphDH subfamilies, as well as PhenDH1, demonstrated significant stabilization of g4-EBNA1 $\left(\Delta T_{\mathrm{m}}=10\right.$ to $\left.20^{\circ} \mathrm{C}\right)$, and other phenanthroline derivatives (PhenDH2-5) demonstrated an even higher stabilization of the substrate $\left(\Delta T_{\mathrm{m}}=20\right.$ to $\left.30^{\circ} \mathrm{C}\right)$, comparable to the result obtained with PhenDC3 $\left(\Delta T_{\mathrm{m}}=30.0^{\circ} \mathrm{C}\right)$. In contrast, all derivatives of the PymDH family, as well as compounds PyDH1 and NaphDH1, demonstrated low or very low stabilization of G4-RNA $\left(\Delta T_{\mathrm{m}}<\right.$ $10^{\circ} \mathrm{C}$ ), illustrating the importance of the nature of heterocyclic residues on the G4-RNA binding properties of ligands. Finally, most derivatives that stabilized g4-EBNA1 also displayed significant level of selectivity with respect to ds-DNA, as their stabilizing effect was almost unaffected by the presence of ds-DNA competitor. The results of the FID assay (Figure 13, C) indicated high g4-EBNA1 affinity (DC 50 $<0.5 \mu \mathrm{M}$ ) for five compounds (PyDH2, PyDH3, and PhenDH1-3), which was comparable to the result obtained for PhenDC3 $\left(D_{50}=0.31 \mu \mathrm{M}\right)$. In contrast, the derivatives PyDH1, PymDH1, PymDH4 and PyDH5 were not able to displace the fluorescent probe (TO) from g4-EBNA1. Of note, none of tested ligands was able to induce displacement of TO from the double-stranded DNA substrate ds 26 ( $\mathrm{DC}_{50}>$ $2.5 \mu \mathrm{M}$ in all cases). 
The systematic comparison of 20 derivatives belonging to the same family allowed us to reveal some relationships between the structure of ligands and their binding to G-quadruplexes in vitro. Thus, the following trend can be deduced with respect to the impact of Het moiety on G4-binding properties in vitro: PhenDH > NaphDH $\approx \mathrm{PyDH}>>\mathrm{PymDH}$. This drastic difference between the subfamily of PymDH derivatives, which did not, or very weakly, bind to G-quadruplexes, and most other derivatives could be rationalized considering the differences in compound geometry imposed by the central heteroaromatic residue, as evidenced by single-crystal structural studies. Indeed, PyDH and PhenDH derivatives adopt $\mathrm{V}$ - and $\mathrm{U}$-shaped conformations, respectively, due to the coordination of water molecules between the two acylhydrazone groups and the central Het residue. Indeed, this conformation is beneficial for binding to G-quadruplex targets, as previously demonstrated in the case of PhenDC3 bound to a parallel-stranded G4-DNA substrate. ${ }^{69}$ In contrast, PymDH derivatives were found to adopt a linear conformation, stabilized by intramolecular bonds between the amide $\mathrm{NH}$ groups and nitrogen atoms of the pyrimidine core. Hence, obviously, this conformation is deleterious for interaction with G-quadruplex targets.

Within each sub-family of ligands, derivatives with lateral pyridinium residues (i.e., PyDH1, PymDH1, NaphDH1, and PhenDH1) systematically demonstrated less efficient binding to G4 structures, in contrast to the derivatives bearing lateral quinolinium heterocycles. Consistently with literature data on related derivatives, ${ }^{70}$ this fact can be attributed to the limited $\pi$-stacking surface of pyridinium derivatives. Conversely, side-chain substituents $(R)$ seem to have little influence on G4-binding properties, even though their impact could not be comprehensively evaluated with our combinatorial matrix.

In summary, the $\mathrm{N}$-acylhydrazone group appeared to be essentially as efficient as the carboxamide group in the design of cationic poly-heteroaromatic $\mathrm{G} 4$ ligands, as can be evidenced from comparison of the results obtained with PhenDC3 and its acylhydrazone analogue PhenDH2 (the former appears as a slightly more efficient binder in fluorescence melting assay, but not in the FID assay). Taken together, these data demonstrate that cationic bis(acylhydrazones) represent a promising scaffold for G4-DNA and G4-RNA binders, providing wide possibilities for further functionalization and modulation of physico-chemical properties.

Biological testing: The effect on EBNA1 expression of the novel derivatives was evaluated using the tests already described above. First, to determine whether this effect was, or not, dependent of the G4-forming GAr-encoding sequence of EBNA1 mRNA, the level of EBNA1 was assessed in H1299 cells transfected with EBNA1 or EBNA1 $\triangle \mathrm{GAr}$ constructs and treated, or not, with all compounds at a $10 \mu \mathrm{M}$ concentration. Interestingly, among the $20 \mathrm{HetDH}$ derivatives, only PyDH2 and PhenDH2 induced GArdependent increase in EBNA1 expression (i.e., they increased EBNA1 level while having no significant effect on EBNA1 $\triangle$ GAr), and three compounds (PyDH3, PymDH5, and PhenDH1) increased EBNA1 level in a GAr-independent manner (i.e., they increased both EBNA1 and EBNA1 $\triangle G A r$ levels). As we were interested in compounds able to interfere with the GAr-dependent limitation of EBNA1 expression and antigen presentation (a mechanism at the basis of EBNA1 immune evasion), we focused only on the compounds that increase EBNA1 level while having no effect on EBNA1 $\triangle G A r$, namely PhenDH2 and PyDH2 (Figure 14). 
A

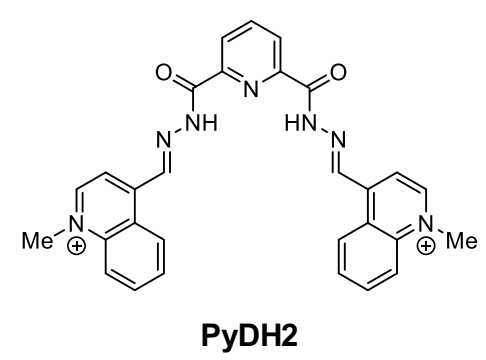<smiles></smiles>

PhenDH2

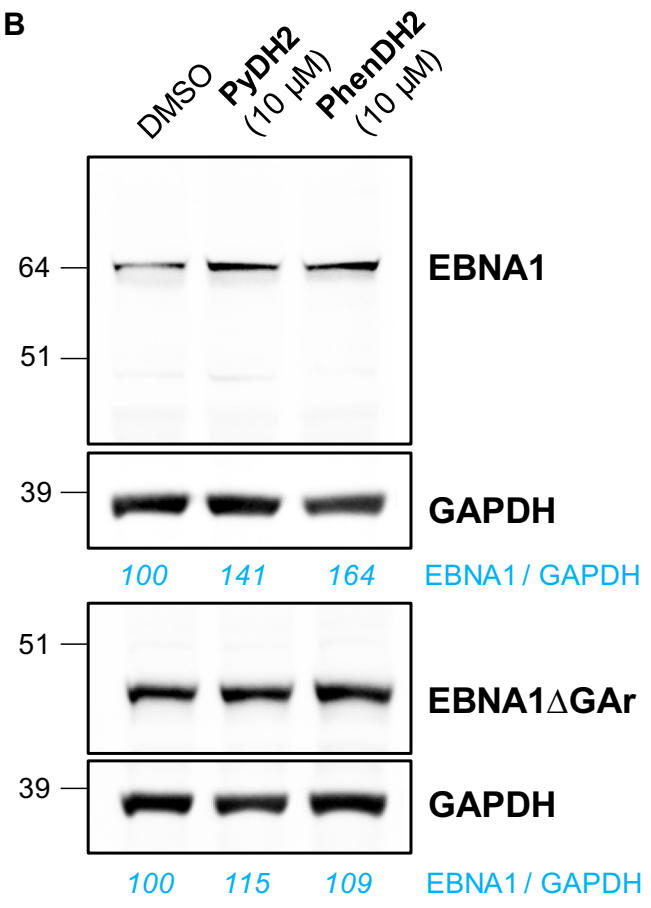

Figure 14. A) Chemical structures of PyDH2 and PhenDH2. B) Expression of EBNA1 (top panel) or EBNA1 $\triangle \mathrm{GAr}$ (bottom panel) in transfected H1299 cells treated with DMSO (control), PyDH2, or PhenDH2 (10 $\mu \mathrm{M}$ each) $40 \mathrm{~h}$ post-transfection. Adapted with permission from Ref. ${ }^{65}$.

Next, we assessed the effect of PyDH2 and PhenDH2 on the GAr-dependent suppression of antigen presentation. In this experiment, $\mathrm{H} 1299$ cells were transfected with murine MHC class I Kb and 235GArOVA or OVA plasmid constructs, treated for $48 \mathrm{~h}$ by the $\mathrm{G} 4$ ligands to be tested and then co-cultured for $24 \mathrm{~h}$ with naive OVA $257-264$ (SL8 peptide)-specific CD8 ${ }^{+}$T-cells from mice. The levels of antigen presentation were determined by measuring the release of IL-2 (a potent growth factor stimulating Tcell proliferation) in the supernatant. IL-2 release increased following the treatment of 235GAr-OVAexpressing cells with compounds PyDH2 or PhenDH2, as compared to DMSO-treated cells (Figure 15). These data indicate that PyDH2 used at a concentration of $10 \mu \mathrm{M}$, as well as PhenDH2 used at a concentration of 5 or $10 \mu \mathrm{M}$ significantly increased the presentation of antigenic peptides from GArOVA (more than a two-fold increase), while having no significant effect on cells expressing OVA. Thus, both compounds interfere with the immune evasion of EBNA1. 
A

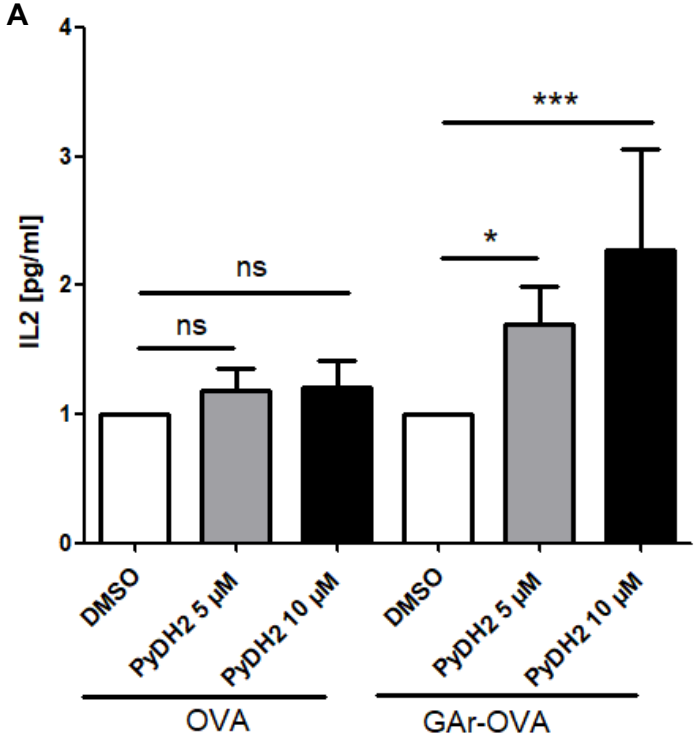

B

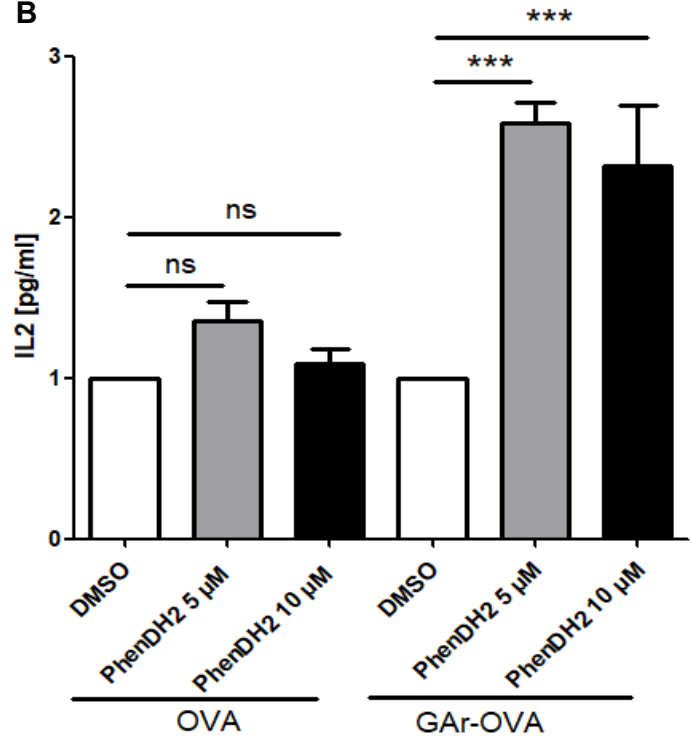

Figure 15. IL2 concentration ( $\mathrm{pg} \mathrm{mL}^{-1}$ ) determined following the treatment of $\mathrm{H} 1299$ cells expressing OVA (left) or GAr-OVA (right) with A) DMSO (control), $5 \mu \mathrm{M}$ and $10 \mu \mathrm{M}$ of PyDH2; B) DMSO (control), $5 \mu \mathrm{M}$ and $10 \mu \mathrm{M}$ of PhenDH2. ${ }^{*}, p<0.05 ;{ }^{* * *}, p<0.001 ;$ ns, not significant. Reproduced with permission from Ref. ${ }^{65}$.

Finally, the toxicity of PyDH2 and PhenDH2 compounds for Mutu-1 (Burkitt's lymphoma) cells in a concentration range of 0.5 to $100 \mu \mathrm{M}$ was assessed using the classical MTT assay. Upon $24 \mathrm{~h}$ treatment, both compounds displayed a relatively low toxicity for Mutu-1 cells (PyDH2: Gl $50>100 \mu \mathrm{M}$, PhenDH2: $50<\mathrm{Gl}_{50}<100 \mu \mathrm{M}$ ). Moreover, the toxicity of both compounds was only slightly higher upon $48 \mathrm{~h}$ treatment (PyDH2: $\mathrm{Gl}_{50} \geq 100 \mu \mathrm{M}$, PhenDH2: $\mathrm{Gl}_{50} \approx 20 \mu \mathrm{M}$ ). Thus, both compounds were not significantly toxic at concentrations which were sufficient to increase the expression of EBNA1 and the production of EBNA1-derived antigenic peptides (i.e., 5-10 $\mu \mathrm{M}$ ), in contrast to PhenDC3, which induced significant toxicity when used at concentrations $>2.5 \mu \mathrm{M}$ in identical conditions. ${ }^{33}$ Thus, as compared to the prototype G4 ligand PhenDC3, novel acylhydrazone derivatives present the advantage of being significantly less toxic.

Mechanistic studies: To verify that PyDH2 and PhenDH2 prevent the interaction of NCL with the G4s of the GAr-encoding sequence of EBNA1 mRNA, we employed the PLA technique described above. Herein, we exploited H1299 cells transiently expressing EBNA1 following transfection with EBNA1 plasmid. Control cells treated with DMSO presented high level of PLA signals (1.83 \pm 0.54 per cell) further confirming that the EBNA1 mRNA-NCL interaction takes place in, or at the close vicinity of the nucleus (Figure 16 A), whereas non-transfected cells did not display PLA signals. Treatment with compounds PyDH2 or PhenDH2 (at a $5 \mu \mathrm{M}$ concentration) significantly reduced both the number $(0.27$ \pm 0.11 and $0.40 \pm 0.21$ per cell, in cells treated with PyDH2 and PhenDH2, respectively) and the intensity of PLA signals, hence confirming the ability of these new derivatives to disrupt the EBNA1 mRNA-NCL interaction. Most importantly, a similar effect was observed in EBV-infected Mutu-1 cells expressing endogenous EBNA1: treatment with $5 \mu \mathrm{M}$ PhenDH2 decreased the average number of PLA signals per cell from $1.68 \pm 0.44$ to $0.59 \pm 0.20$ (Figure 16, B).

Finally, using the RNA pull-down assay described above and based on the use of biotin-tagged oligonucleotide g4-EBNA1 and recombinant NCL, we observed that PyDH2 or PhenDH2 (at $10 \mu \mathrm{M}$ ) reduced the amount of RNA-bound NCL almost 10-fold with respect to DMSO control; in identical 
conditions, PhenDC3 employed at the same concentration reduced the amount of bound NCL only two-fold. ${ }^{33}$ Collectively, these results strongly suggest that PyDH2 and PhenDH2, similarly to but more efficiently than PhenDC3, act by preventing NCL to interact with G4s that form in the GAr-encoding sequence of EBNA1 mRNA.
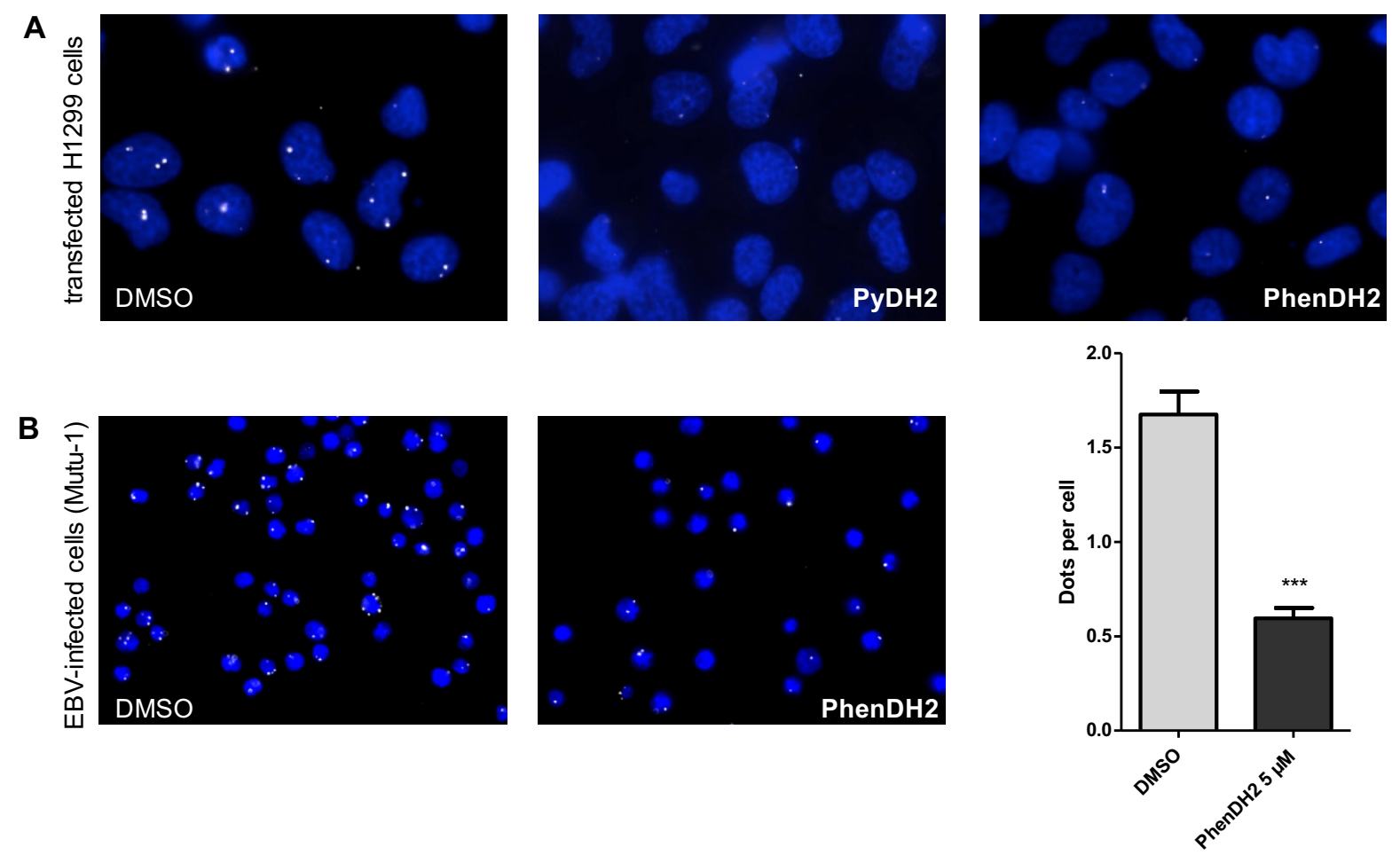

Figure 16. A) Proximity ligation assay (PLA) performed in $\mathrm{H} 1299$ cells transiently expressing EBNA1. Microscopy images of cells treated with DMSO (control), PyDH2 $(5 \mu \mathrm{M})$, and PhenDH2 (5 $\mu \mathrm{M})$. Nuclei appear in blue (DAPI staining); white dots (PLA signals) indicate the interaction between NCL and EBNA1 mRNA.-B) Same experiments performed in EBV-infected Mutu-1 cells endogenously expressing EBNA1, treated by DMSO (control) or treated with PhenDH2 (5 $\mu \mathrm{M})$. Right panel: number of nuclear PLA signals (dots) per cell in Mutu-1 cells treated with DMSO (control) or with PhenDH2 $(5 \mu \mathrm{M})$. Data from two biological replicates ( $\geq 200$ cells per sample); $* * *, p<$ 0.001. Adapted with permission from Ref. ${ }^{65}$.

The systematic comparison of HetDH derivatives allowed us to reveal several interesting relationships between the structure of ligands, their binding to G-quadruplexes in vitro, and their ability to act as modulators of EBNA1 expression in cells. Thus, while most derivatives of $\mathrm{PyDH}, \mathrm{NaphDH}$, and PhenDH subfamilies were found to bind quadruplexes with high affinity in vitro, only two derivatives, PyDH2 and PhenDH2, increased EBNA1 expression in $\mathrm{H} 1299$ cells in a GAr-dependent manner. Interestingly, four naphthyridine derivatives (NaphDH2-5) demonstrated significant stabilization of g4-EBNA1, according to fluorescence melting experiments (Figure 13, B), but had not influence on EBNA1 expression. This behavior is not without precedent, as it was also observed with pyridostatin (PDS), and indicates that ligand-induced stabilization of a G4 structure is not sufficient to predict its capacity to compete with NCL for $\mathrm{G} 4$ binding. In fact, one can imagine that stabilization of G4 structure could even favor their interaction with NCL (cf. Figure 16). Hence, G4 stabilization and ability to interfere with NCL-G4 interaction may be two independent events. In line, all $\mathrm{NaphDH}$ derivatives were significantly less active in displacing the fluorescence probe TO from g4-EBNA1 ( $\left.D_{50}>0.5 \mu \mathrm{M}\right)$, in contrast to the two biologically active derivatives, PyDH2 and PhenDH2. This also indicates that FID 
assay is potentially better suited for the first-line selection of G4-targeting drugs acting through interference with protein binding to $\mathrm{G} 4$ structures.

One final remark addresses the G4-RNA vs. G4-DNA selectivity of G4 ligands. Indeed, PhenDC3 as well as PyDH2 and PhenDH2 also bind G4-DNA in vitro, independently of the folding topology of the latter. In this regard, the EBNA1-specific cellular activity of the two compounds can be attributed to the fact that GAr repeat of EBNA1 mRNA contains a cluster of multiple G4-forming sequences, which may be particularly susceptible to ligand-induced effects such as NCL displacement. Moreover, G4-DNA structures form only transiently during DNA transactions such as replication, transcription and recombination, and are otherwise disfavored in the double-stranded DNA context; this is not the case with RNA whose single-stranded nature favors the formation of long-lived secondary motifs such as G4.

\section{Conclusions}

There is currently no specific treatment for most EBV-related cancers, therefore it is of high significance and importance to develop more targeted therapies that exploit specific aspects of EBV biology. The integrative chemical biology approach described in this chapter, based on chemical genetics in yeast, cellular biology of EBV in mammalian cells, and combined with chemistry of G4 ligands, has allowed to collect a large body of data, which altogether constitute the proof of concept for a new strategy to unveil EBV-related cancers to the immune system, and thereby to fight against EBV infection. This strategy is based on the interaction between the host protein nucleolin (NCL) and the G4 structures formed in GAr-encoding sequence of the viral EBNA1 mRNA. As deciphered in this work, the disruption of this NCL/G4-RNA interaction alleviates the EBNA1 self-inhibition of translation, thereby stimulating neoantigen presentation. This finding, completely novel from the mechanistic viewpoint, led to identification of the NCL/G4 association as a relevant and druggable target to unveil EBV-infected cells to the host immune system. A first striking feature of this ensemble of data is the connection between the length-dependent effect of the Gly-Ala repeat (GAr) on the inhibition of its own translation and antigenic peptides presentation on one side, and the GAr-encoding sequence of EBNA1 mRNA ability to form multiple clustered quadruplex structures on the other side. Making a parallel between these two observations sheds light on the crucial role of the NCL/G4 mRNA association and validates the model of G4-mediated inhibition of EBNA1 translation, if one assumes that the longer the length of GAr, the higher is the number of G4 motifs, providing a higher number of NCL binding sites. These mechanistic findings allow to make a step further in understanding of G4-RNA functions by showing that these secondary structures can serve as recruiting platform for factors regulating mRNA translation, and not only as roadblock-like repressors. ${ }^{30}$ Despite the fact that several proteins are known to interact with G4-RNA, so far none of them has been reported to regulate mRNA translation through direct interaction with $\mathrm{G} 4$, which emphasizes the novel role of NCL unraveled in this work. ${ }^{31}$

On the ground of these observations, we identified G4-interactive compounds as potential candidates for disrupting the $\mathrm{G} 4 / \mathrm{NCL}$ interaction, which is the critical molecular event at the basis of our strategy. PhenDC3, the first hit compound, belongs to the "bisquinolinium" class of G4-interacting ligands that exhibit high G4-binding affinity and selectivity. The ability PhenDC3 to compete with NCL for binding to G4 of EBNA1 mRNA has been demonstrated both in vitro and in cellulo and is fully consistent with its capacity to act as competitive inhibitor of NCL for binding G4-DNA substrates as reported recently. ${ }^{42}$ Subsequently a new collection of compounds based on a DiHydrazone (DH), instead of DiCarboxamide 
(DC) scaffold has been generated. The synthetic strategy stems on a combinatorial approach that yielded $20 \mathrm{HetDH}$ derivatives exhibiting structural diversity with regard to the central aromatic core and lateral heterocyclic moieties. This diversity combined with our set of in vitro and in cellulo assays enabled establishing firm structure activity relationships (SAR) to identify the most promising candidates. Taken together our results demonstrate that, i) the acylhydrazone linker does not significantly impart the $\mathrm{G} 4$ binding properties as compared with carboxamide analogues, ii) the binding of $\mathrm{G} 4$ structures is mostly governed by the nature of the central and lateral heterocyclic residues and the resulting preorganization ( $\mathrm{V}$ - or U- vs. linear shape) of the chemical scaffold. Interestingly we found that there is no systematic correlation between in vitro G4-binding features and in cellulo activity, which has been already noted with the benchmark compound PDS. ${ }^{33}$ This trend once again highlights the need to introduce as early as possible in cellulo evaluation assays in the workflow for selecting robust lead compounds. In line, two new compounds PyDH2 and PhenDH2 have been selected for their promising biological properties and patent-protected. ${ }^{71}$ In fact, these were shown to be able to efficiently disrupt the NCL/EBNA1-G4 interaction in cells, to interfere with the GAr dependent inhibition of EBNA1 expression and to promote the GAr-restricted antigen presentation (Figure 17). These two new derivatives are also significantly less cytotoxic than the prototype compounds PhenDC3. Globally, our approach of medicinal chemistry and cellular biology led to the rapid selection of new promising drugs candidates with improved in cellulo properties and better therapeutic index. Of note, the comparison of the different activities through our in vitro to in cellulo test pipeline is of importance to determine if the compounds operate through the same molecular mechanism or not (e.g., some compounds may interfere with EBNA1 production in a negative way, or disrupt NCL/G4 mRNA interaction without stimulating the antigenic presentation, or vice versa). In addition, the fact that some G4 ligands, despite binding EBNA1 mRNA G4 with high affinity, are unable to prevent the binding of NCL on these G4 indicate that different mode of binding exist and that only some of them could interfere with NCL binding. The way by which the active G4 ligands prevent NCL binding can itself involve various mechanism. It can be a direct competition (i.e., NCL and the G4 ligand share the same, or at least overlapping, binding site(s) on G4). G4 ligands may also favor, or stabilize, a particular G4 structure which is poorly recognized by NCL. Hence, in addition to their interest for further developments toward the clinic, the active G4 ligands we have already isolated represent interesting chemical biology tools to explore this issue.
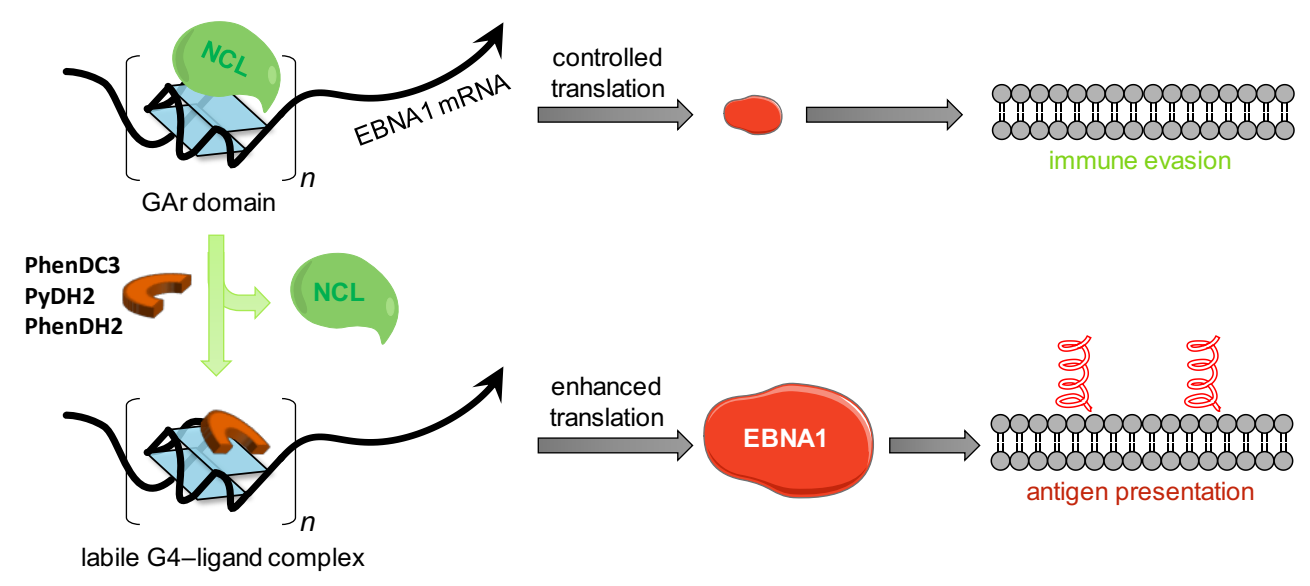

Figure 17. Schematic representation of the mechanism of immune evasion of EBV and the effect of nucleolindisplacing $\mathrm{G} 4$ ligands. 
From the therapeutic perspective, the reported strategy is without precedent and steps on a firm mechanistic rationale for discovery of drug candidates towards emergence of clinical solutions for the specific treatment of EBV-related cancers. Indeed it is essential to mention that overcoming GAr-based self-inhibition of EBNA1 translation should unveil EBV-carrying tumor cells to cytotoxic T cells without having significant effect on the vast majority of healthy host cells, as most tumor cells from EBV-related cancers are infected by EBV whereas, in healthy individuals, the latent infection by EBV is primarily restricted to a specific small pool of memory $B$ cells. Finally, our data shed light on the complex interplay between the viral protein EBNA1, its mRNA and the host cell factors, and thus open the door to entirely new EBV-specific therapeutic approaches.

From a more general perspective, interfering with specific protein-G4 RNA complexes holds promise for new therapeutic development as well as for furthering our understanding of G4-related biological processes. Therefore, it would be of utmost interest to explore the generality of this G4-RNA/NCLmediated immune evasion. Indeed, on one hand G4 motifs have been found in a number of pathogens and could be possibly used to escape immune system detection using various mechanisms, ${ }^{47,72-74}$ and, on the other hand, NCL has been reported to be capable of interfering with a number of virus by acting on different phases of the infection..$^{29,75-77}$ More specifically, other oncogenic herpesviruses, like the Kaposi sarcoma-associated herpesvirus (KSHV), have evolved similar strategy of G4 clustering in the coding regions of their genome maintenance proteins (GMPs) to evade immune system. ${ }^{78,79}$ Hence, our strategy and the new G4-interacting compounds identified in our work may find applications as therapeutic avenues for the treatment of other gamma-herpesvirus infections and related diseases.

\section{Acknowledgements}

The authors wish to thank all the collaborators involved in this work for their invaluable contribution. This work was supported by Agence Nationale de Recherche (grant-in aid ANR-17-CE07-0004-01 to AG), La Ligue contre le Cancer (to RF), La Ligue contre le Cancer -CSIRGO (grant 2017-18 to MB), Institut National du Cancer (grant 2016-169 to MB, RF, and MPTF), and Fondation pour la Recherche Médicale (grant DCM20181039571 to MB, RF, and MPTF).

\section{References}

1. Epstein, M. A.; Achong, B. G.; Barr, Y. M. Lancet 1964, 283, 702-703.

2. Damania, B. Nat. Rev. Microbiol. 2004, 2, 656-668.

3. Young, L. S.; Rickinson, A. B. Nat. Rev. Cancer 2004, 4, 757-768.

4. $\quad$ Cohen, J. I.; Fauci, A. S.; Varmus, H.; Nabel, G. J. Sci. Transl. Med. 2011, 3, $107 f$ 7 7.

5. Daskalogianni, C.; Pyndiah, S.; Apcher, S.; Mazars, A.; Manoury, B.; Ammari, N.; Nylander, K.; Voisset, C.; Blondel, M.; Fåhraeus, R. J. Pathol. 2015, 235, 334-341.

6. Kanakry, J. A.; Ambinder, R. F. Curr. Treat. Options Oncol. 2013, 14, 224-236.

7. Wang, M.; Jiang, S.; Liu, X.; Wang, Y. Appl. Microbiol. Biotechnol. 2013, 97, 6251-6262.

8. Wang, M.; Jiang, S.; Han, Z.; Zhao, B.; Wang, L.; Zhou, Z.; Wang, Y. Appl. Microbiol. Biotechnol. 2016, 100, 1221-1230.

9. Wilson, J.; Manet, E.; Gruffat, H.; Busson, P.; Blondel, M.; Fahraeus, R. Cancers 2018, 10, 109. 
10. Norseen, J.; Johnson, F. B.; Lieberman, P. M. J. Virol. 2009, 83, 10336-10346.

11. Shastri, N.; Schwab, S.; Serwold, T. Annu. Rev. Immunol. 2002, 20, 463-493.

12. Cardinaud, S.; Starck, S. R.; Chandra, P.; Shastri, N. PLoS One 2010, 5, e8692.

13. Levitskaya, J.; Coram, M.; Levitsky, V.; Imreh, S.; Steigerwald-Mullen, P. M.; Klein, G.; Kurilla, M. G.; Masucci, M. G. Nature 1995, 375, 685-688.

14. Yin, Y.; Manoury, B.; Fåhraeus, R. Science 2003, 301, 1371-1374.

15. Tellam, J.; Connolly, G.; Green, K. J.; Miles, J. J.; Moss, D. J.; Burrows, S. R.; Khanna, R. J. Exp. Med. 2004, 199, 1421-1431.

16. Apcher, S.; Daskalogianni, C.; Manoury, B.; Fåhraeus, R. PLoS Pathog. 2010, 6, e1001151.

17. Fåhraeus, R. Nat. Rev. Mol. Cell Biol. 2005, 6, 263-267.

18. Apcher, S.; Komarova, A.; Daskalogianni, C.; Yin, Y.; Malbert-Colas, L.; Fåhraeus, R. J. Virol. 2009, 83, 1289-1298.

19. Tellam, J. T.; Lekieffre, L.; Zhong, J.; Lynn, D. J.; Khanna, R. PLoS Pathog. 2012, 8, e1003112.

20. Tellam, J. T.; Zhong, J.; Lekieffre, L.; Bhat, P.; Martinez, M.; Croft, N. P.; Kaplan, W.; Tellam, R. L.; Khanna, R. PLoS Pathog. 2014, 10, e1004423.

21. Norseen, J.; Thomae, A.; Sridharan, V.; Aiyar, A.; Schepers, A.; Lieberman, P. M. EMBO J. 2008, 27, 3024-3035.

22. Murat, P.; Zhong, J.; Lekieffre, L.; Cowieson, N. P.; Clancy, J. L.; Preiss, T.; Balasubramanian, S.; Khanna, R.; Tellam, J. Nat. Chem. Biol. 2014, 10, 358-364.

23. Bugaut, A.; Alberti, P. Biochimie 2015, 113, 125-133.

24. Amrane, S.; Adrian, M.; Heddi, B.; Serero, A.; Nicolas, A.; Mergny, J.; Phan, A. T. J. Am. Chem. Soc. 2012, 134, 5807-5816.

25. Piazza, A.; Adrian, M.; Samazan, F.; Heddi, B.; Hamon, F.; Serero, A.; Lopes, J.; Teulade-Fichou, M.-P.; Phan, A. T.; Nicolas, A. EMBO J. 2015, 34, e201490702.

26. Jodoin, R.; Bauer, L.; Garant, J.-M.; Mahdi Laaref, A.; Phaneuf, F.; Perreault, J.-P. RNA 2014, 20, 1129-1141.

27. Angrand, G.; Quillévéré, A.; Loaëc, N.; Daskalogianni, C.; Granzhan, A.; Teulade-Fichou, M.-P.; Fahraeus, R.; Prado Martins, R.; Blondel, M. Genes 2019, 10, 667.

28. Zimmer, J.; Tacconi, E. M. C.; Folio, C.; Badie, S.; Porru, M.; Klare, K.; Tumiati, M.; Markkanen, E.; Halder, S.; Ryan, A.; Jackson, S. P.; Ramadan, K.; Kuznetsov, S. G.; Biroccio, A.; Sale, J. E.; Tarsounas, M. Mol. Cell 2016, 61, 449-460.

29. Tosoni, E.; Frasson, I.; Scalabrin, M.; Perrone, R.; Butovskaya, E.; Nadai, M.; Palù, G.; Fabris, D.; Richter, S. N. Nucleic Acids Res. 2015, 43, 8884-8897.

30. Beaudoin, J.-D.; Perreault, J.-P. Nucleic Acids Res. 2010, 38, 7022-7036.

31. Millevoi, S.; Moine, H.; Vagner, S. Wiley Interdiscip. Rev. RNA 2012, 3, 495-507. 
32. Cammas, A.; Millevoi, S. Nucleic Acids Res. 2017, 45, 1584-1595.

33. Lista, M. J.; Martins, R. P.; Billant, O.; Contesse, M.-A.; Findakly, S.; Pochard, P.; Daskalogianni, C.; Beauvineau, C.; Guetta, C.; Jamin, C.; Teulade-Fichou, M.-P.; Fåhraeus, R.; Voisset, C.; Blondel, M. Nat. Commun. 2017, 8, 16043.

34. Lista, M. J.; Martins, R. P.; Angrand, G.; Quillévéré, A.; Daskalogianni, C.; Voisset, C.; TeuladeFichou, M.-P.; Fåhraeus, R.; Blondel, M. Microb. Cell 2017, 4, 305-307.

35. Voisset, C.; Daskalogianni, C.; Contesse, M.-A.; Mazars, A.; Arbach, H.; Le Cann, M.; Soubigou, F.; Apcher, S.; Fåhraeus, R.; Blondel, M. Dis. Model. Mech. 2014, 7, 435-444.

36. Ginisty, H.; Sicard, H.; Roger, B.; Bouvet, P. J. Cell Sci. 1999, 112, 761-772.

37. Hanakahi, L. A.; Sun, H.; Maizels, N. J. Biol. Chem. 1999, 274, 15908-15912.

38. González, V.; Guo, K.; Hurley, L.; Sun, D. J. Biol. Chem. 2009, 284, 23622-23635.

39. González, V.; Hurley, L. H. Biochemistry 2010, 49, 9706-9714.

40. Lago, S.; Tosoni, E.; Nadai, M.; Palumbo, M.; Richter, S. N. Biochim. Biophys. Acta - Gen. Subj. 2017, 1861, 1371-1381.

41. Dickerhoff, J.; Onel, B.; Chen, L.; Chen, Y.; Yang, D. ACS Omega 2019, 4, 2533-2539.

42. Saha, A.; Duchambon, P.; Masson, V.; Loew, D.; Bombard, S.; Teulade-Fichou, M.-P. Biochemistry 2020, in press.

43. Gomez, D.; Shankman, L. S.; Nguyen, A. T.; Owens, G. K. Nat. Methods 2013, 10, 171-177.

44. Martins, R. P.; Findakly, S.; Daskalogianni, C.; Teulade-Fichou, M.-P.; Blondel, M.; Fåhraeus, R. Molecules 2018, 23, 3124.

45. Iborra, F. J. Science 2001, 293, 1139-1142.

46. Al-Jubran, K.; Wen, J.; Abdullahi, A.; Roy Chaudhury, S.; Li, M.; Ramanathan, P.; Matina, A.; De, S.; Piechocki, K.; Rugjee, K. N.; Brogna, S. RNA 2013, 19, 1669-1683.

47. Martins, R. P.; Malbert-Colas, L.; Lista, M. J.; Daskalogianni, C.; Apcher, S.; Pla, M.; Findakly, S.; Blondel, M.; Fåhraeus, R. Nucleic Acids Res. 2019, 47, 3086-3100.

48. Piazza, A.; Boulé, J.-B.; Lopes, J.; Mingo, K.; Largy, E.; Teulade-Fichou, M.-P.; Nicolas, A. Nucleic Acids Res. 2010, 38, 4337-4348.

49. Prorok, P.; Artufel, M.; Aze, A.; Coulombe, P.; Peiffer, I.; Lacroix, L.; Guédin, A.; Mergny, J.-L.; Damaschke, J.; Schepers, A.; Ballester, B.; Méchali, M. Nat. Commun. 2019, 10, 3274.

50. Halder, K.; Largy, E.; Benzler, M.; Teulade-Fichou, M.-P.; Hartig, J. S. ChemBioChem 2011, 12, 1663-1668.

51. Bugaut, A.; Balasubramanian, S. Nucleic Acids Res. 2012, 40, 4727-4741.

52. Gray, L. T.; Puig Lombardi, E.; Verga, D.; Nicolas, A.; Teulade-Fichou, M.-P.; Londoño-Vallejo, A.; Maizels, N. Cell Chem. Biol. 2019, 26, 1681-1691.e5.

53. Sun, Z.-Y.; Wang, X.-N.; Cheng, S.-Q.; Su, X.-X.; Ou, T.-M. Molecules 2019, $24,396$. 
54. Maleki, P.; Mustafa, G.; Gyawali, P.; Budhathoki, J. B.; Ma, Y.; Nagasawa, K.; Balci, H. Nucleic Acids Res. 2019, 47, 10744-10753.

55. Yangyuoru, P. M.; Di Antonio, M.; Ghimire, C.; Biffi, G.; Balasubramanian, S.; Mao, H. Angew. Chem. Int. Ed. 2015, 54, 910-913.

56. Liu, X.; Ishizuka, T.; Bao, H.-L.; Wada, K.; Takeda, Y.; lida, K.; Nagasawa, K.; Yang, D.; Xu, Y. J. Am. Chem. Soc. 2017, 139, 7533-7539.

57. Largy, E.; Hamon, F.; Rosu, F.; Gabelica, V.; De Pauw, E.; Guédin, A.; Mergny, J.-L.; TeuladeFichou, M.-P. Chem. Eur. J. 2011, 17, 13274-13283.

58. Thota, S.; Rodrigues, D. A.; Pinheiro, P. de S. M.; Lima, L. M.; Fraga, C. A. M.; Barreiro, E. J. Bioorg. Med. Chem. Lett. 2018, 28, 2797-2806.

59. Fraga, C.; Barreiro, E. Curr. Med. Chem. 2006, 13, 167-198.

60. Tributino, J.; Santos, M.; Mesquita, C.; Lima, C.; Silva, L.; Maia, R.; Duarte, C.; Barreiro, E.; Fraga, C.; Castro, N.; Miranda, A.; Guimaraes, M. Br. J. Pharmacol. 2010, 159, 1716-1723.

61. Rodrigues, D. A.; Ferreira-Silva, G. A.; Ferreira, A. C. S.; Fernandes, R. A.; Kwee, J. K.; SantAnna, C. M. R.; Ionta, M.; Fraga, C. A. M. J. Med. Chem. 2016, 59, 655-670.

62. Rollas, S.; Küçükgüzel, S. Molecules 2007, 12, 1910-1939.

63. Maia, R. do C.; Tesch, R.; Fraga, C. A. M. Expert Opin. Ther. Pat. 2014, 24, 1161-1170.

64. Alam, M.; Verma, G.; Shaquiquzzaman, M.; Marella, A.; Akhtar, M.; Ali, M. J. Pharm. Bioallied Sci. 2014, 6, 69.

65. Reznichenko, O.; Quillévéré, A.; Martins, R. P.; Loaëc, N.; Kang, H.; Lista, M. J.; Beauvineau, C.; González-García, J.; Guillot, R.; Voisset, C.; Daskalogianni, C.; Fåhraeus, R.; Teulade-Fichou, M.P.; Blondel, M.; Granzhan, A. Eur. J. Med. Chem. 2019, 178, 13-29.

66. Daina, A.; Michielin, O.; Zoete, V. Sci. Rep. 2017, 7, 1-13.

67. Muegge, I.; Heald, S. L.; Brittelli, D. J. Med. Chem. 2001, 44, 1841-1846.

68. Daina, A.; Zoete, V. ChemMedChem 2016, 1117-1121.

69. Chung, W. J.; Heddi, B.; Hamon, F.; Teulade-Fichou, M.-P.; Phan, A. T. Angew. Chem. Int. Ed. 2014, 53, 999-1002.

70. Dhamodharan, V.; Harikrishna, S.; Jagadeeswaran, C.; Halder, K.; Pradeepkumar, P. I. J. Org. Chem. 2012, 77, 229-242.

71. Blondel, M.; Quillevéré, A.; Voisset, C.; Lista, M. J.; Fahraeus, R.; Daskologianni, C.; PradoMartins, R.; Teulade-Fichou, M.-P.; Granzhan, A.; Beuavineau, C.; Reznichenko, O. Novel hydrazone derivatives for preventing or treating EBV-related cancers. WO/2019/193071, 2019.

72. Harris, L. M.; Merrick, C. J. PLOS Pathog. 2015, 11, e1004562.

73. Ruggiero, E.; Richter, S. N. Nucleic Acids Res. 2018, 46, 3270-3283.

74. Bian, W.-X.; Xie, Y.; Wang, X.-N.; Xu, G.-H.; Fu, B.-S.; Li, S.; Long, G.; Zhou, X.; Zhang, X.-L. Nucleic Acids Res. 2019, 47, 56-68. 
75. Grinstein, E.; Wernet, P.; Snijders, P. J. F.; Rösl, F.; Weinert, I.; Jia, W.; Kraft, R.; Schewe, C.; Schwabe, M.; Hauptmann, S.; Dietel, M.; Meijer, C. J. L. M.; Royer, H.-D. J. Exp. Med. 2002, 196, 1067-1078.

76. Muller, M.; Hutin, S.; Marigold, O.; Li, K. H.; Burlingame, A.; Glaunsinger, B. A. PLOS Pathog. 2015, 11, e1004899.

77. Perrone, R.; Butovskaya, E.; Lago, S.; Garzino-Demo, A.; Pannecouque, C.; Palù, G.; Richter, S. N. Int. J. Antimicrob. Agents 2016, 47, 311-316.

78. Sorel, O.; Dewals, B. G. Front. Microbiol. 2019, 9, 3315.

79. Dabral, P.; Babu, J.; Zareie, A.; Verma, S. C. J. Virol. 2020, in press. 